\title{
Probabilistic Defense and Impact of Generator Ramp Constraints Induced Load Redistribution Attacks in Power Systems
}

\author{
Kommoju C Sravanthi and Mercy Rosalina Kotapuri* \\ Department of Electrical and Electronics Engineering, Vignan's Foundation for Science, Technology and Research, Vadlamudi, Guntur, \\ Andhra Pradesh, India.
}

Received 7 December 2020; Accepted 2 April 2021

\begin{abstract}
Despite today's smart grid's deployment of more information and communication systems, it is more prone to data injection attacks. Researchers have developed several methodologies for successful intrusion into the system by means of False Data Injection Attacks (FDIAs) which result in system vulnerability. Practical FDIAs are called Load Redistribution Attacks (LRAs). LRAs target bus active power injections and line active power flows. The most damaging Load Redistribution Attack Vector (LRAV) of a classical LRA is found by solving a Bilevel Programming Problem (BPP) where attacker in the upper level develops most damaging LRAV and operator in the lower level performs basic static Security Constrained Optimal Power Flow (SCOPF) along with false measurements. But in dynamic operations, generator ramp rates play a crucial role. So, in this article, generator ramp rate constraints are included in SCOPF to show the impact of Generator Ramp Rate Constraints induced LRA (GRC-LRA) in means of Economic Loss, \$/MWh, without attacking any generator. GRC-LRA vector is solved by using Karush-Kuhn-Tucker (KKT) based single level Mixed Integer Linear Programming Problem (MILPP) and Benders Decomposition methods. An optimal attack-defense strategy is also found by playing static zero-sum game, a method of probabilistic defense. Attack and defense mechanisms for GRC-LRA are tested on modified IEEE-14 bus test system and validated at fixed and variable ramp rates in low load varying and high load varying conditions.
\end{abstract}

Keywords: Load Redistribution Attack (LRA); Generator Ramp Constraints induced Load Redistribution Attack (GRC-LRA); Bilevel Programming Problem (BPP); KKT based single level MILPP Method; Benders Decomposition Method; Probabilistic defense; Static Zero-Sum Game;

\section{Introduction}

Modern power systems is a fundamental asset in the development of every nation's economy, reliability and security. Smart grid advancements in power grid have made the power system not only physical but also cyber. CyberPhysical Power System (CPPS) is complex as the cyber layer covers every part of the physical layer by sensing, communicating and processing to operate Supervisory Control and Data Acquisition (SCADA) system in a better way [1]. Even though CPSS has many advancements, it is vulnerable to cyber threats/attacks due to its wide usage of information and communication systems [2]. It is a real experience to Ukrainian power system, by coming across such cyber attacking through a pre-installed malware by opening breakers autonomously [3]. So it is a fact to get aware of the effect of cyber-threats on the power systems.

Various researchers have developed many proposals and more literature to develop, analyze and defend cyber-attacks [4]. CPSS' components can be hacked or their keys can be cracked to disrupt the grid, furthermore smart measuring units can be compromised, to create man-in-middle attacks [5], to build Denial-of-Service (DoS) attacks [6] by injecting false/bad data into smart units. To detect false/bad data injected into the system, classical bad data detection methods $\left(\chi^{2}\right.$-distribution hypothesis testing) were been used by the operator at control center. But Liu et al. have proposed that

*E-mail address: kmr_eee@vignan.ac.in

ISSN: 1791-2377 @ 2021 School of Science, IHU. All rights reserved.

doi:10.25103/jestr.143.15 there is a chance to bypass those classical detection methods and intrude into the system by injecting undetectable false data into the system and named that attack called False Data Injection Attack (FDIA) and that specific attack vector is False Data Injection Attack Vector (FDIAV) [7]. FDIAs divert the operator to make erroneous decisions due to incorrect state estimates caused by FDIAVs.

FDIA that especially targets bus active power injection measurement values and line active power flow measurement values is popular as Load Redistribution Attack (LRA) and that injected vector is Load Redistribution Attack Vector (LRAV). These practical FDIAs (LRAs) were proposed by Yuan et al. to deteriorate the system economics and reliability [8]. Generally, basic detection methods are based on residual analysis, i.e., if the residue of actual and estimated measurements is within tolerance, then as per $\chi^{2}$ - hypothesis testing, the measurement doesn't have bad data. If tolerance value is low, then the capability of detecting bad data can be high. So, if an attack vector is within tolerance then perhaps the attacking values cannot be detected. It should be also noted that an attack vector can be undetectable, if mean of change in errors is zero. Hence a successful LRAV can be developed such that the sum of load changes must be equal to zero. In other means, actual load should be redistributed among the loads. Hence, this attack is named as LRA. LRAVs were developed based on Kirchoff's laws [8]. LRAs severely cause load shedding, economic loss and line outages too. LRAVs are of two types one is Immediate LRA [8] and other is Delayed LRA [9]. LRAs are generally solved by framing Bilevel Programming Problem (BPP) solved by Karush- 
Kuhn-Tucker (KKT) conditions based single level MILPP [10] or by Benders decomposition [9]. Liu et al. have approximated the load shedding and economic loss on solving two levels individually within less time [11]. Choeum and Choi have proposed a new type LRA in three-phase distribution system in which BPP is solved by KKT based single-level MILPP and results are validated on IEEE-13 node distribution feeder [12]. Kaviani and Hedman have developed a new structure of LRA problem, mentioned that attackers view can be trivial and used physical laws on transmission line flows (by making the sum of change in line flows equals to zero) to build a successful LRAV [13].

More research is done on various types of attacks like local LRAs [14, 15], cyber-physical coordinated LRAs [1618], cascading failures coordinated with LRAs [19] and also the reliability analysis after a successful LRA [20, 21]. Shayan and Amraee developed LRAs in conjunction with Security Constrained Unit Commitment (SCUC) problem (with ramp constraints, minimum up and down times). To lessen LRAV's loss, Cyber Secured Unit Commitment (CSUC) problem has also been developed [22]. Dae-Hyun Choi and Le Xie have proposed a special type ramp induced data attacks that target the real-time market by using look-ahead SCOPF dispatch model. Initial generation dispatches are disrupted by attacker. Obviously ramp rate constraints (dependent on initial dispatches) can get deviated from actuals which result in financial arbitrage in the market. In this type of attack, attacker needs to compromise the generator's measuring unit [23]. Che et al. have developed a special type of attack called "Ramp Induced Data Attacks" which create power imbalance (either surplus/deficient power generation) by attacking a generator but not loads [24]. From literature, it should be noted that generator ramp rates can provide assistance for an attacker to maximize his/her objectives and it is needed to concentrate on ramp constraints integrated attacks. Xiang and Wang proposed that an optimal attack-defense strategy can be found by probabilistic based game theory, where critical measuring units are selected based on critical operating points in the system [26] or entropic degree [27]. This method of selecting may not be applicable all time if any load changes occur.

In this article, the impact of ramp rates of a generator is shown in means of economic loss without attacking a generator but attacking loads/lines using LRA. This is achieved by inducing ramp rate constraints along with LRA on basic SCOPF problem, proposed as Generator Ramp Constraints induced Load Redistribution Attack (GRC-LRA). It should be noted that in the proposed GRC-LRA, no generator is attacked. In the upper-level of GRC-LRA's BPP, an LRAV is developed for given load/line attacking resources and in the lower level of dynamic SCOPF (basic SCOPF and generator ramp rates) contributes to deviated uneconomic generation dispatches.

Major Contributions of this research article are as follows:

1. Framing the overall BPP of GRC-LRA to find the most damaging GRC-LRAV and solving it by KKT conditions based single-level MILLPP and Benders decomposition methods.

2. Finding the critical measuring units and obtaining the probabilities of an optimal attack-defense strategy of GRC-LRAV using static zero-sum game theory.

3. Exhibiting a numerical analysis to show the impact of GRC-LRAV on a modified IEEE-14 bus test system with low load changing and high load changing at fixed, variable ramp rates and protection against GRC-LRA.
This research article is organized in a way that section 2 deals with literature of FDIAs, LRAs and modelling of ramp constraints in SCED/SCOPF whereas section 3 presents a BPP to find most damaging GRC-LRAV, two methods to solve that BPP by using KKT based single-level MILPP and Benders decomposition methods and probabilistic defense static zero-sum game theory. Section 4 depicts a numerical analysis of LRAV versus GRC-LRAV applied on modified IEEE-14 bus test system in two load varying conditions at fixed and variable ramp rates. Moreover, Section 4 also represents probabilities of optimal attack-defense strategies and finally conclusions are described in section 5 .

\section{Background}

\subsection{False Data Injection Attacks (FDIAs) and Load Redistribution Attack (LRA)}

Power system online monitoring system is more prone to undetectable false data that can be injected into the network measurement devices like Phasor Measurement Units (PMUs)/Remote Terminal Units (RTUs) which are used for measuring, controlling and observing the system time-to-time [25]. Generally, sensed real-time active and reactive power injections and line flows are sent to the control center by means of communication and security equipment like Intelligent Electronic Devices (IEDs), firewalls and WANs. At the control center various analysis like DC state estimation, SCOPF/SCED, load forecasting, contingency analysis and Energy Marketing System (EMS) marketing will be undergone. Undetectable FDIAs, developed by Liu et al. in 2011 makes system vulnerable. If $\boldsymbol{z}, \boldsymbol{a}$ and $\boldsymbol{z}_{\boldsymbol{a}}$ are the actual, attack and damaged/attacked measurement vectors respectively. Let $\widehat{\boldsymbol{x}}$ be the actual state estimate of $\boldsymbol{z}, \widehat{\boldsymbol{x}}_{\boldsymbol{f}}$ be the false state estimate of $\boldsymbol{z}_{\boldsymbol{a}}=\boldsymbol{z}+\boldsymbol{a}, \quad c$ be the reflected erroneous estimate of $\boldsymbol{z}_{\boldsymbol{a}}$ and $\boldsymbol{H}$ be the Jacobian matrix, then the $L_{2}$ norm of the residual with damaged measurements is $\left\|\boldsymbol{z}_{\boldsymbol{a}}-\boldsymbol{H} * \widehat{\boldsymbol{x}}_{\boldsymbol{f}}\right\|$ is simplified as shown in Eq.1.

$$
\begin{aligned}
& \text { But } \widehat{x}_{\boldsymbol{f}}=\widehat{\boldsymbol{x}}+\boldsymbol{c} \text {, } \\
& \text { then }\left\|z_{a}-\boldsymbol{H} * \widehat{\boldsymbol{x}}_{\boldsymbol{f}}\right\|=\left\|\mathbf{z}_{\boldsymbol{a}}-\boldsymbol{H} *(\widehat{\boldsymbol{x}}+\boldsymbol{c})\right\| \\
& \left.\Rightarrow\left\|z_{a}-H * \widehat{x}_{f}\right\|=\| z+a-H \widehat{x}-H c\right) \| \\
& \Rightarrow\left\|z_{a}-H * \widehat{x}_{f}\right\|=\|(z-H \widehat{x})+(a-H c)\|
\end{aligned}
$$

If $\boldsymbol{a}=\boldsymbol{H} \boldsymbol{c}, L_{2}$-norm of attacked measurement residual becomes $L_{2}$-norm of non-attacked measurement residual [7]. Hence, it can be clear that $L_{2}$-norm of damaged measurement residual is also within the threshold and it is obvious that $\boldsymbol{z}_{\boldsymbol{a}}$ can bypass classical detection method and disrupt the system. The attack vector $\boldsymbol{z}_{\boldsymbol{a}}$ is called FDIAV.

A practical example of FDIA is LRA. In LRA the attacker targets are bus active power injections' and line active power flows' measurements. Load Redistribution Attack Vector (LRAV), the name itself defines that load must be redistributed among loads and that vector can be undetectable if the sum of redistributed load is zero (as per Gaussian distribution mean of errors is zero). Redistribution among loads should be done within a tolerance $\pm \tau$, so that LRAV can be undetectable by control center [8].

Attacker always seeks to maximize the operating cost with minimum number of resources and tries to develop a most damaging undetectable LRAV within the available resources abided to his/her constraints at one-time step. In the next time step the operator tries to minimize operating cost subjected to basic SCOPF constraints like supply-load 
balance, power flow constraints, and generator, line and load curtailment bounds. This problem can be framed as a BPP where in the upper level attacker maximizes and in the lower level operator minimizes the operating cost. The bilevel representation to obtain a most damaging undetectable LRAV is given in Fig. 1 [8].

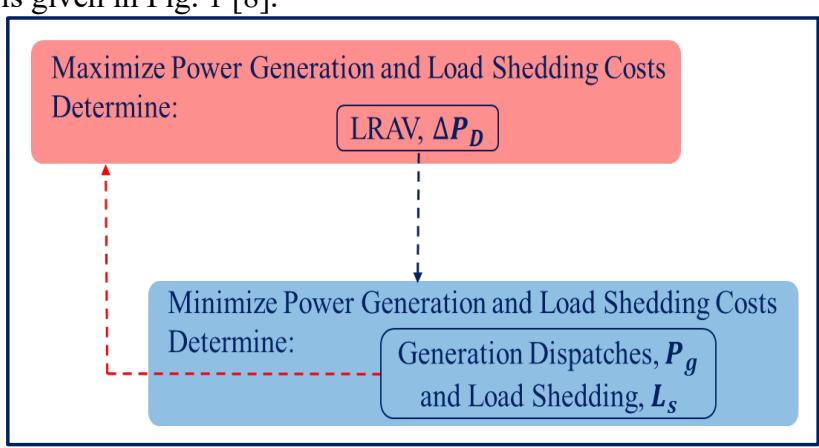

Fig. 1. Bilevel Representation of LRA

The mathematical representation of a most damaging LRAV's BPP is given by Eq.2-Eq.14, where upper level replicates attacker and lower level denotes operator. Objective functions of upper level, Eq.2 and lower level, Eq.9 are maximization and minimization of generational operational and load shedding costs respectively. Eq.3 and Eq. 4 show that sum of load redistributed must be zero and load change at particular node must be within tolerance $\pm \tau$, Eq.5 gives the changes in line flows due to LRAV, however Eq.6 and Eq.7 deal with attacker compromising the measurements and Eq.8 shows the number of attackable resources. Furthermore, Eq.10-Eq.14 are the basic SCOPF constraints and their bounds.

$$
\begin{aligned}
& \operatorname{Max}_{\Delta \boldsymbol{P}_{\boldsymbol{D}}} \sum_{i=1}^{N_{g}} C_{g_{i}} * P_{g_{i}}^{*}+\sum_{k=1}^{N_{d}} C_{s_{k}} * L_{s_{k}}^{*} \\
& \text { s.t. } \sum_{k=1}^{N_{d}} \Delta P_{D_{k}}=0 \\
& -\tau P_{D_{k}} \leq \Delta P_{D_{k}} \leq \tau P_{D_{k}} \\
& \Delta \boldsymbol{P}_{L}=-S F . K D \cdot \Delta \boldsymbol{P}_{D} \\
& \Delta P_{D_{k}}=0 \Leftrightarrow \theta_{D_{k}}=0 \Longrightarrow \\
& \int \begin{array}{l}
\Delta P_{D_{k}}+\tau P_{D_{k}} \theta_{D_{k}} \geq 0 \\
\Delta P_{D_{k}}-\tau P_{D_{k}} \theta_{D_{k}} \leq 0
\end{array} \\
& \theta_{D+_{k}}+\theta_{D-_{k}}-2 \theta_{D_{k}} \leq 0 \\
& \left\{\Delta P_{D_{k}}+\left(-\tau P_{D_{k}}-\varepsilon\right) \theta_{D+_{k}} \geq-\tau P_{D_{k}}\right. \\
& \Delta P_{D_{k}}+\left(\tau P_{D_{k}}+\varepsilon\right) \theta_{D-_{k}} \leq \tau P_{D_{k}} \\
& \theta_{D++_{k}}+\theta_{D-_{k}}+\theta_{D_{k}} \leq 2 \\
& \theta_{D++_{k}}+\theta_{D-k}-\theta_{D_{k}} \geq 0 \\
& \theta_{D++_{k}}, \theta_{D-{ }_{k}}, \theta_{D_{k}} \in\{0,1\}
\end{aligned}
$$

$$
\Delta P_{L_{l}}=0 \Leftrightarrow \theta_{L_{l}}=0 \Rightarrow\left\{\begin{array}{c}
\Delta P_{L_{l}}+M \theta_{L_{l}} \geq 0 \\
\Delta P_{L_{l}}-M \theta_{L_{l}} \leq 0 \\
\theta_{L+l}+\theta_{L-l}-2 \theta_{L_{l}} \leq 0 \\
\Delta P_{L_{l}}+(-M-\varepsilon) \theta_{L+l} \geq-M \\
\Delta P_{L_{l}}+(M+\varepsilon) \theta_{L-l} \leq M \\
\theta_{L+l}+\theta_{L-l}+\theta_{L_{l}} \leq 2 \\
\theta_{L++_{l}}+\theta_{L-l}-\theta_{L_{l}} \geq 0 \\
\theta_{L+l^{\prime}}, \theta_{L-l}, \theta_{L_{l}} \in\{0,1\}
\end{array}\right.
$$

$\sum_{k=1}^{N_{d}} \theta_{D_{k}}+2 \sum_{l=1}^{N_{l}} \theta_{L_{l}} \leq R_{t}$

$\left\{\boldsymbol{P}_{\boldsymbol{g}}{ }^{*}, \boldsymbol{L}_{\boldsymbol{s}}{ }^{*}\right\}=\operatorname{Min}_{\boldsymbol{P}_{\boldsymbol{g}}, \boldsymbol{L}_{\boldsymbol{s}}} \sum_{i=1}^{N_{g}} C_{g_{i}} * P_{g_{i}}^{*}+\sum_{k=1}^{N_{d}} C_{s_{k}} * L_{s_{k}}^{*}$

$\sum_{i=1}^{N_{g}} P_{g_{i}}=\sum_{k=1}^{N_{d}}\left(P_{D_{k}}-L_{s_{k}}\right)$

$$
P_{L}=S F . K P . P_{g}-S F . K D .\left(P_{D}+\Delta P_{D}-L_{s}\right)
$$

$-P_{L_{l}}^{\max } \leq P_{L_{l}} \leq P_{L_{l}}^{\max }$

$P_{g_{i}}^{\min } \leq P_{g_{i}} \leq P_{g_{i}}^{\max }$

$0 \leq L_{s_{k}} \leq P_{D_{k}}+\Delta P_{D_{k}}$

\subsection{Ramp Constraints in SCOPF/SCED}

Practically, generators may take time to reach a new level from the operating point especially in case of steam and hydrothermal generating units (due to their inner dynamics) which is called ramping. Generally, ramping in generators is of three types: startup, shutdown and operating ramp constraints. Startup and shutdown ramping constraints exist when a decommitted generator is committed and a committed generator is decommitted correspondingly. When a unit is committed from off position, the generator reaches its minimum operating capacity by increasing gradually within some time periods is startup ramping. If a generator is to be decommitted to off position, the generator reaches it minimum operating capacity by decreasing gradually within some time periods is shutdown ramping. These two ramping constraints come into existence in unit commitment problem. But in economic dispatch operating ramp constraints are considered which are neither startup ramping nor shutdown ramping constraints. Operating ramping deals with the generation dispatches of two successive time periods subjected to generator bounds. Let $P_{g_{i}}(t)$ and $P_{g_{i}}(t-1)$ be the dispatches of $i^{t h}$ generator at time steps of $t$ and $t-1$. Let $R_{-} u_{g_{i}}$ and $R_{-} d_{g_{i}}$ be the ramp rates for up and down ramping of $i^{t h}$ generator and $\Delta t=(t)-(t-1)$ be time step size then ramp up (Eq.15) and ramp down (Eq.16) constraints, as functions of generation power dispatches are as follows:

$P_{g_{i}}(t)-P_{g_{i}}(t-1) \leq R \_u_{g_{i}}+P_{g_{i}}^{\min }$ 
$P_{g_{i}}(t-1)-P_{g_{i}}(t) \leq R_{-} d_{g_{i}}+P_{g_{i}}^{\min }$

$\forall i \in 1,2, \ldots, N_{g}$ and $\forall t \in 1,2, \ldots, T$.

Eq.15 and Eq.16 approximate that $i^{\text {th }}$ generator may be able to step up or down from the operating point within the ramp up and down limits respectively, but not beyond.

\section{Generator Ramp Constraints Induced LRA (GRC-}

\section{LRA)}

The main aim of this research is to show the impact of ramp constraints on the system when an attacker is intruded into the system and able to develop a successful LRAV. As in practice, for high rating generating units, ramp constraints are mandate and hence ramp constraints need to be included while analyzing LRAs. So to find most damaging Generator Ramp Constraints Induced Load Redistribution Attack Vector (GRC-LRAV), the incorporation of ramp constraints at upper or lower level of BPP must be known. It is an assumption in LRAs that generators cannot be attacked [8] and in this article no generator is attacked. So, inclusion of ramp constraints in the upper level is not possible however it is needed to include ramp up and ramp down constraints in the lower level of BPP i.e., in SCED/SCOPF. Hence the overall problem of most damaging GRC-LRAV is as follows, given by Eq.17-Eq.20:

$$
\begin{aligned}
& \underset{\Delta P_{D}}{\operatorname{Max}} \sum_{i=1}^{N_{g}} C_{g_{i}} * P_{g_{i}}^{*}+\sum_{k=1}^{N_{d}} C_{s_{k}} * L_{s_{k}}^{*} \\
& \text { s.t. (3) - (8) } \\
& \left\{P_{g}{ }^{*}, L_{s}^{*}\right\}=\operatorname{Min}_{P_{g}, L_{s}} \sum_{i=1}^{N_{g}} C_{g_{i}} * P_{g_{i}}^{*}+\sum_{k=1}^{N_{d}} C_{s_{k}} * L_{s_{k}}^{*} \\
& \text { s.t. (10) }-(14),(15),(16)
\end{aligned}
$$

The BPP to find most damaging LRAV with induced generator ramp constraints (i.e., GRC-LRAV) can be solved by various methods. Basic method is like converting the BPP into single level MILPP using KKT conditions or dual conditions and thereafter solving single level MILPP by using MILPP solvers [8-10]. Other method is solving BPP directly by one of the decomposition methods like Benders decomposition [9]. In this article, most damaging GRCLRAV is obtained by solving using KKT conditions based single-level MILPP and Benders decomposition methods.

\subsection{Most Damaging GRC-LRAV by KKT Conditions based MILPP}

The most damaging GRC-LRAV is obtained by converting upper and lower levels of GRC-LRA's BPP into single level MILPP using KKT conditions are given below. The overall objective function is maximization of operational cost subjected to upper level constraints Eq.3-Eq.8, lower level constraints Eq.10-Eq.14 and Eq.9 is replaced by new equations with new variables Eq.21-Eq.30.

$$
\begin{aligned}
& \underset{\Delta P_{D}}{\operatorname{Max}} \sum_{i=1}^{N_{g}} C_{g_{i}} * P_{g_{i}}^{*}+\sum_{k=1}^{N_{d}} C_{s_{k}} * L_{s_{k}}^{*} \\
& \text { s.t. (18), (20) }
\end{aligned}
$$$$
-\vartheta_{l}-\underline{\mathrm{A}}_{l}+\overline{\mathrm{A}}_{l}=0
$$

$$
\begin{aligned}
& c_{i}-\lambda+\left(S F . K P_{i}\right)^{T} . \vartheta-\underline{B}_{i}+\overline{\mathrm{B}}_{i}+r u_{i}^{t}-r u_{i}^{(t+1)}+r d_{i}^{t}+ \\
& r d_{i}^{(t+1)}=0 ; \forall t \epsilon 1,2, \ldots, T-1
\end{aligned}
$$

$$
\begin{aligned}
& c_{i}-\lambda+\left(S F . K P_{i}\right)^{T} \cdot \vartheta-\underline{B}_{i}+\bar{B}_{i}+r u_{i}^{t}-r d_{i}^{t}=0 ; \forall t= \\
& T
\end{aligned}
$$

$C S_{k}-\lambda+\left(S F . K D_{k}\right)^{T} \cdot \vartheta-\underline{\Gamma}_{k}+\bar{\Gamma}_{k}=0$

$\underline{\mathrm{A}}_{l}, \overline{\mathrm{A}}_{l}, \underline{\mathrm{B}}_{i}, \overline{\mathrm{B}}_{i}, \underline{\Gamma}_{k}, \bar{\Gamma}_{k} \geq 0$

$$
\left\{\begin{array}{c}
\underline{\mathrm{A}}_{l} \leq M \omega_{\underline{A}, l} \\
P_{L_{l}}+P_{L_{l}}^{\max } \leq M\left(1-\omega_{\underline{\mathrm{A}}, l}\right) \\
\overline{\mathrm{A}}_{l} \leq M \omega_{\overline{\mathrm{A}}, l} \\
P_{L_{l}}^{\max }-P_{L_{l}} \leq M\left(1-\omega_{\overline{\mathrm{A}}, l}\right) \\
\omega_{\underline{\mathrm{A}, l},}+\omega_{\overline{\mathrm{A}, l}} \leq 1
\end{array}\right.
$$

$$
\left\{\begin{array}{c}
\underline{\mathrm{B}}_{i} \leq M \omega_{\underline{\mathrm{B}}, i} \\
P_{g_{i}}-P_{g_{i}}^{\min } \leq M\left(1-\omega_{\underline{\mathrm{B}}, i}\right) \\
\overline{\mathrm{B}}_{i} \leq M \omega_{\overline{\mathrm{B}}, i} \\
P_{g_{i}}^{\max }-P_{g_{i}} \leq M\left(1-\omega_{\overline{\mathrm{B}, i}}\right) \\
\omega_{\underline{\mathrm{B}}, i}+\omega_{\overline{\mathrm{B}, i}} \leq 1
\end{array}\right.
$$

$$
\left\{\begin{array}{c}
\Gamma_{k} \leq M \omega_{\bar{\Gamma}, k} \\
L_{s_{k}} \leq M\left(1-\omega_{\underline{\Gamma}, k}\right) \\
\bar{\Gamma}_{k} \leq M \omega_{\overline{\Gamma, k}} \\
P_{D_{k}}+\Delta P_{D_{k}}-L_{s_{k}} \leq M\left(1-\omega_{\overline{\Gamma, k}}\right) \\
\omega_{\overline{\Gamma, k}}+\omega_{\overline{\Gamma, k}} \leq 1
\end{array}\right.
$$

$$
\left\{\begin{array}{c}
r u_{i}^{t} \leq M \omega_{r u_{i}^{t}} \\
P_{g_{i}}(t-1)-P_{g_{i}}(t)+R_{-} u_{i}+P_{g_{i}}^{\min } \leq M\left(1-\omega_{r u_{i}^{t}}\right) \\
r d_{i}^{t} \leq M \omega_{r d_{i}^{t}} \\
P_{g_{i}}(t)-P_{g_{i}}(t-1)+R_{-} d_{i}+P_{g_{i}}^{\min } \leq M\left(1-\omega_{r d_{i}^{t}}\right) \\
\omega_{r u_{i}^{t}}+\omega_{r d_{i}^{t}} \leq 1
\end{array}\right.
$$

$\forall t \in 1,2, \ldots, T$

$$
\omega_{\underline{\mathrm{A}}, l}, \omega_{\overline{\mathrm{A}, l},}, \omega_{\underline{\mathrm{B}}, i}, \omega_{\overline{\mathrm{B}, i}}, \omega_{\underline{\Gamma}, k}, \omega_{\overline{\Gamma, k}, k}, \omega_{r u_{i}^{t}}, \omega_{r d_{i}^{t}} \in\{0,1\}
$$

$\forall l \epsilon 1,2, \ldots, N_{l}, \forall i \epsilon 1,2, \ldots, N_{g}, \forall k \epsilon 1,2, \ldots, N_{d}$, 
Eq.21-Eq.30 are KKT based necessary feasibility constraints and Eq.26-Eq.29 are obtained by complementary slackness conditions.

\subsection{Most Damaging GRC-LRAV by Benders} Decomposition.

Benders decomposition algorithm has the capability to decompose an integer programming or MILPP into master problem and sub problem(s) which can be solved separately by iterative process. General Benders decomposition method when used to find worst GRC-LRAV may result in local optima. So, it is better to use multi-start Benders decomposition algorithm to obtain global optima of most damaging GRC-LRAV [9].

The Master Problem (MP) of GRC-LRA's BPP is given by equation Eq.31:

$\operatorname{Min}_{\Delta P_{D}}-\alpha$

s.t. (18)

$-C_{g_{i}} P_{g_{i}}^{*}-c s_{k} L_{s_{k}}^{*}-\boldsymbol{\mu}^{(m-1, n-1) *}$

$\left(\Delta \boldsymbol{P}_{\boldsymbol{D}}-\left(\Delta \boldsymbol{P}_{\boldsymbol{D}}^{(m-1, n-1)}\right) \leq \alpha\right.$ if $m>1$

$-C_{g_{i}} P_{g_{i}}-c s_{k} L_{s_{k}}^{*}-\boldsymbol{\mu}^{(\text {last mat } n-1, n-1) *}$

$\left(\Delta \boldsymbol{P}_{\boldsymbol{D}}-\Delta \boldsymbol{P}_{\boldsymbol{D}}^{(\text {last mat } n-1, n-1)}\right) \leq \alpha$ if $n>1$

And the Sub Problem (SP) of GRC-LRA's BPP is given by Eq.32

$$
\begin{gathered}
\operatorname{Min}_{P_{g}, L_{s}} \sum_{i=1}^{N_{g}} C_{g_{i}} * P_{g_{i}}^{*}+\sum_{k=1}^{N_{d}} C_{s_{k}} * L_{s_{k}}^{*} \\
\text { s.t. (20) } \\
\Delta P_{D_{k}}-\Delta P_{D_{k}}^{(m, n)}=0
\end{gathered}
$$

Let the global optima of BPP be $z_{\text {opt }}$, Benders loops iteration counter be $m$ and the Benders restarting counter be $n$. The procedure to solve GRC-LRA's BPP by multi start Benders decomposition is given as follows:

1. Initialize $\mathrm{n}=1, \mathrm{~m}=1$ and $z_{\text {opt }}=\infty$.

2. Solve MP and update $\Delta P_{D_{k}}=\Delta P_{D_{k}}^{(m, n)}$ and $z_{l o}^{(m, n)}=$ $\alpha^{(m, n)}$.

3. Solve SP and update $P_{g}^{(m, n)}, L_{s}^{(m, n)}$ and

$$
\begin{aligned}
& Z_{u p}^{(m, n)} \\
& =\left\{\begin{array}{c}
\min \left\{\sum_{i=1}^{N_{g}} C_{g_{i}} P_{g_{i}}^{(m, n)}+\sum_{k=1}^{N_{d}} C_{s_{k}} L_{s_{k}}^{(m, n)}\right\} \begin{array}{c}
\text { if } m=1 \\
\&
\end{array}=1 \\
\min \left\{\sum_{\text {up }}^{(m-1, n)} C_{g_{i}} P_{g_{i}}^{(m, n)}+\sum_{k=1}^{N_{d}} C_{s_{k}} L_{s_{k}}^{(m, n)}\right\} \text { if } m>1 \\
\min \left\{Z_{u p}^{(\text {last } m \text { at } n-1, n-1)}{ }^{N_{g}} C_{g_{i}} P_{g_{i}}^{(m, n)}+\sum_{k=1}^{N_{d}} C_{s_{k}} L_{s_{k}}^{(m, n)}\right\} \text { if } m=1 \\
\& n \neq 1
\end{array}\right.
\end{aligned}
$$

4. To avoid the global optima not to stuck at local optimal $\Delta P_{D_{k}}$ need to be updated as:

$$
\left\{\begin{array}{c}
\text { if } \Delta P_{D_{k}}^{(m, n)}=\tau P_{D_{k}}^{(m, n)}, \text { and } \mu_{k}^{(m, n)}>0 \text {, then set } \mu_{k}^{(m, n)}=0 \\
\text { if } \Delta P_{D_{k}}^{(m, n)}=-\tau P_{D_{k}}^{(m, n)}, \text { and } \mu_{k}^{(m, n)}<0 \text {, then set } \mu_{k}^{(m, n)}=0
\end{array}\right.
$$

5. Update global optima as $z_{o p t}=z_{u p}^{(m, n)}$ if $z_{u p}^{(m, n)}<$ $z_{\text {opt }}$.

6. Check the convergence criteria $\left|z_{u p}^{(m, n)}-z_{l o}^{(m, n)}\right|<$ $\varepsilon$. If "Yes" then go to step-10, else go to step-7.

7. If $z_{u p}^{(m, n)}-z_{l o}^{(m, n)}>0$, update $m=m+1$ and go to step-2.

8. If $z_{u p}^{(m, n)}-z_{l o}^{(m, n)}<0$, go to step-9.

9. Update $n=n+1$, go to step-2 (neglect $\mu^{(m, n)}$ of all previous iterations except the last iteration $\left.\mu^{(m, n-1)}\right)$.

\section{Stop}

The flowchart of solving GRC-LRAV by Benders decomposition method is shown in Fig. 2.

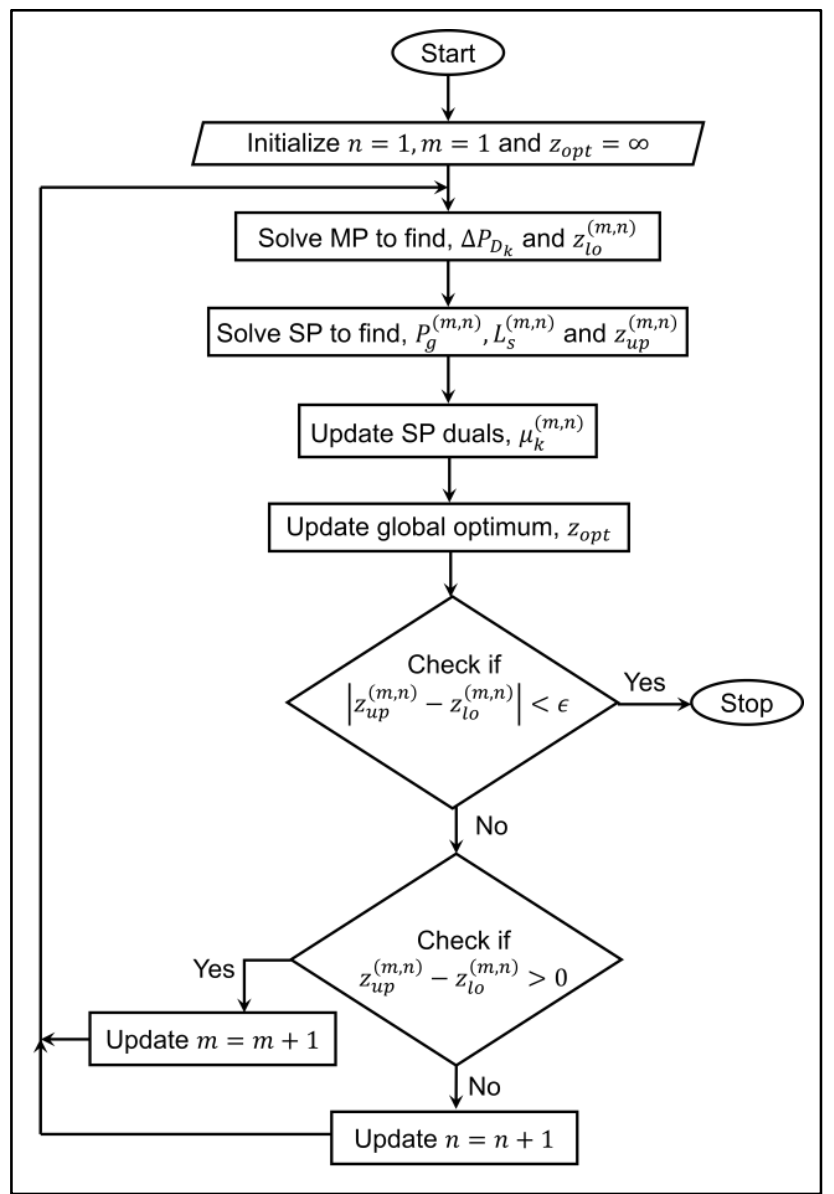

Fig. 2. Flowchart to obtain most damaging GRC-LRAV by Benders decomposition

\subsection{Probabilistic Defense Against GRC-LRAV:}

Various analysis regarding LRAs or GRC-LRAs is carried out to find an optimal defense mechanism against attacks as attacker tries to maximize the economic loss and defender targets to minimize it [26, 27]. So certain optimal cyberdefense must be provided such that attacker cannot inject a 
successful LRAV into the system even if he/she intrudes. An LRAV can become unsuccessful/detectable, if $\sum_{m=1}^{k} \Delta P_{D_{m}} \neq$ 0 . If any of the critical measuring units is not attackable (means defended), then that unit won't help the attacker to develop a successful LRAV. Attacker may not have access to all measuring units and defender also can't defend all units (may be due to budget issues). So, certain critical measuring units must be considered which have high rank in attacking. If any unit has high rank of attacking, then that unit is highly probable for defending. That unit can be called as a critical measuring unit. It is also not an optimal strategy to protect only one unit all time. To obtain optimal defense, attack or defense strategies should be changing. Hence it can be derived that the units must have some probability for attacking or defending. In the literature, critical measuring units are selected based on entropic degree [27] and units operating at critical points [26]. These methods are deficient if the load changes.

To select an optimal number of critical measurements for prior known load and given attack resources, a method can be followed as follows:

Step-1: Apply defense to the measuring unit of $k^{\text {th }}$ load or $l^{\text {th }}$ line i.e., $\theta_{D_{k}}=\{\}$ or $\theta_{L_{l}}=\{\}$ and find economic loss where \{\} represents null set.

Step-2: Find economic loss to all units where one unit is defended each time.

Step-3: Select the units which give minimum economic loss $\cong 0$ and treat them as critical units.

It is to be noticed that this method is applicable if the approximate load demand is known prior to both attacker and defender and the maximum approximate number of attacker resources should also be known.

Hence to find an optimal attack-strategy, probabilistic based game theory framework can be used. This game has two players, attacker and defender. Attacker tries to maximize his/her utility and defender minimize his/her utility. The utility function in this game is economic loss. Attacker and defender have their own strategies (actions) and are independent. Hence this game is a static game. It is considered that attacker and defender spends unit cost for attacking or defending [26]. Perhaps, an optimal attack-defense strategy can be obtained from a Nash equilibrium point by playing a static zero-sum game. Nash equilibrium is an equilibrium point for both players which says that any player moving from this point may not get extra incentives. In this problem, Nash equilibrium (optimal attack-defense strategy) results in pure probability of one attack-one defense strategy or mixed probabilities of possible attack or defense strategies [26]. Based on the Nash equilibrium, it is found that which attack strategy is highly probable and based on that defense can be provided by the operator.

\section{Numerical Example}

The main aim of the article is to show the impact of generator ramp constraints induced into LRA's BPP model i.e., to show the economic loss created by most damaging GRC-LRAV and also to obtain optimal attack-defense strategy considering economic loss as utility. Sections 4.1. and 4.2. show the analysis in two scenarios namely fixed ramp rates and variable ramp rates. In each scenario (in each section of 4.1. and 4.2.), it is again considered that the load variations are low and high. Low load variations are given in sub-sections4.1.1. and 4.2.1. and high load variations are given in 4.1.2. and 4.2.2. Moreover, it is also analyzed in a way that if fixed ramp rates are slack (not-tight) or tight, are demonstrated in subsections 4.1.1. and 4.1.2. whereas, variable ramp rates as slack and tight in low load and high load changes are analyzed in subsections 4.2.1.1., 4.2.1.2., 4.2.2.1. and 4.2.2.2 respectively. Section 4.3. shows the selection of critical measuring units and probabilities of optimal attack-defense strategies.

In this research article, the test case considered is modified IEEE-14 bus system where data is obtained from MATPOWER [28]. Modified IEEE-14 bus system has five generators, one zero injection bus, eleven load buses and twenty transmission lines. As per the assumptions in LRA, it is clear that generators and zero injection buses can't be attacked. However, the attackable measuring units are one measuring unit at each load bus and two measuring units for each line. Hence the maximum number of attackable resources be $11+2 * 20=51$ for a modified IEEE-14 bus system. For analysis, it is considered that the maximum power transfer capabilities of the first, second and remaining lines are $160 \mathrm{MW}, 70 \mathrm{MW}$ and $60 \mathrm{MW}$ respectively. It is also considered that the attacker may be capable to attack 20 measuring units. CPLEX solvers are used to solve the MILPPs and LPPs in this work [29].

Basically in look-a-head market, generation dispatches are calculated for all hours of next day whereas in real-time market, generator dispatches are calculated for every 10-15 minutes. So the step size in look-a-head market is high and which means that the load change can be large and similarly in real-time market step size is comparatively low where load change is also low. At the two loading conditions the load changes from $t-1$ to $t$ is low and the other is the load change from $t-1$ to $t$ is high. It is considered that for analysis in high load variations, the number of time instances be 24 and in low load variations the number is 6 . In this research, the ramp rates, $R_{-} u_{g_{i}}$ and $R_{-} d_{g_{i}}$ are considered as fixed and variable in all time instances. It is also assumed that the attacker might not attack all time during all instances. He/she also tries to induce the LRAV at the instant when the load is high or the load change is high, so that the attacker can maximize his/her profit.

Most damaging GRC-LRAV of the test system in low load and high load variations' scenarios at fixed/variable ramp rates is found by using two methods explicitly KKT conditions based single level MILPP and Benders decomposition methods. The number of variables used to solve Eq.18, Eq.20, Eq.21-Eq.30 by KKT conditions based single level MILPP and to solve MP (Eq.31) and SP (Eq.32) by Benders decomposition respectively, are given in Tab.1.

\subsection{Economic Loss due to Most Damaging GRC-LRAV at Fixed Ramp Rates}

\subsubsection{Scenario-1: Low Load Variation with $T=6$ at Slack and Tight Fixed Ramp Rates}

Let the load variations within six time instances and the attack triggering are as given in Tab.2. For better analysis of GRCLRAV, it is considered that the ramp rates are slack (not-tight)

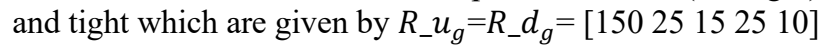

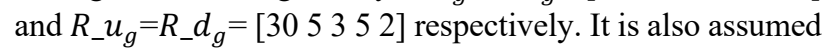
that generator dispatches at $0^{\text {th }}$ instant are $P_{g}^{(0)}=\left[\begin{array}{lll}230 & 5.41\end{array}\right.$ $\left.\begin{array}{lll}14.33 & 0 & 8.27\end{array}\right]$ where the considered initial load demand is $258 \mathrm{MW}$. 
Kommoju C Sravanthi and Mercy Rosalina Kotapuri/

Journal of Engineering Science and Technology Review 14 (3) (2021) 124 - 139

Table 1. Parameters to obtain most damaging GRC-LRAV of a modified IEEE-14 bus test system

\begin{tabular}{c|c|c|c|c}
\hline \multirow{2}{*}{ Parameter } & \multicolumn{2}{|c|}{$\begin{array}{c}\text { KKT Conditions based single level } \\
\text { MILPP }\end{array}$} & \multicolumn{2}{c}{ Benders Decomposition } \\
\cline { 2 - 5 } & Low Load Change & High Load Change & Low Load Change & High Load Change \\
\hline Variables Number & 2070 & 8280 & 961 & 3841 \\
Equalities' Number & 468 & 1872 & 318 & 1272 \\
Inequalities' Number & 3162 & 12648 & 1933 & 7729 \\
Integer Constrained & 1050 & 4200 & 558 & 2232 \\
Variables & & & & \\
\hline
\end{tabular}

Table 2. Low load variation and attack triggers with $T=6$

\begin{tabular}{c|c|c|c|c|c|c}
\hline Time Instances & 1 & 2 & 3 & 4 & 5 & 6 \\
\hline Load & 259 & 254 & 260 & 260 & 253 & 259 \\
$\boldsymbol{R}$ & 0 & 0 & 20 & 0 & 0 & 20 \\
\hline
\end{tabular}

Most damaging GRC-LRAV, at low load varying conditions i.e., analogous to real-time market operations, applied on test system is obtained by solving KKT conditions based single level MILPP and Benders decomposition method. As the variable size is high (as shown in Tab.1), it is not possible to represent all solved variables. So, generation dispatches $\left(P_{g}\right)$, load shedding $\left(L_{s}\right)$, attack vector $\left(\Delta P_{D}\right)$ and operational cost are represented in Tab. 3 and Tab.4.

$P_{g}, L_{s}$ and $\Delta P_{D}$ satisfying all constraints of GRC-LRAV at low load variation within 6 time instances with tight ramp rates are shown in Tab.3. If attack is not triggered, irrespective of ramp rates, load shedding is zero. At $3^{\text {rd }}$ and $6^{\text {th }}$ instances attack is triggered. Hence, in case of LRA, total load shedding is $26.3421 \mathrm{MW}$ whereas in case of GRC-LRA, total load shedding is $40.5788 \mathrm{MW}$ as shown in Tab.3. It is to be noted that in this research, generator is not attacked (only loads and lines are attacked), so the attack vector, $\Delta P_{D}$ in case of LRA and GRC-LRA is same as shown in the $3^{\text {rd }}$ and $4^{\text {th }}$ rows of Tab.3.

Total operational cost and load shedding at low load variation at fixed tight ramp rates in 6 time steps of LRA and GRC-LRA are given in Tab.4.

Table 3. Generator power dispatches, $P_{g}$ and attack vector, $\Delta P_{D}$ in LRA and GRC-LRA at tight fixed ramp rates in low load variation

\begin{tabular}{|c|c|c|c|c|c|c|c|c|}
\hline \multirow{2}{*}{\multicolumn{2}{|c|}{ 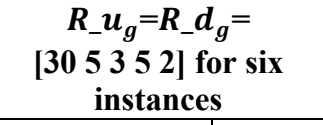 }} & \multirow{3}{*}{$\begin{array}{c}\text { Bus } \\
\text { No. } \\
1\end{array}$} & \multicolumn{6}{|c|}{ Time Instances } \\
\hline & & & 1 & 2 & 3 & 4 & 5 & 6 \\
\hline \multirow{6}{*}{ LRA } & \multirow{5}{*}{$P_{g}$} & & 230.00 & 230.00 & 196.52 & 230.00 & 230.00 & 196.14 \\
\hline & & 2 & 5.47 & 5.14 & 0.00 & 5.54 & 5.07 & 0.00 \\
\hline & & 3 & 14.67 & 12.94 & 30.00 & 15.02 & 12.60 & 30.00 \\
\hline & & 6 & 0.00 & 0.00 & 0.00 & 0.00 & 0.00 & 0.00 \\
\hline & & 8 & 8.85 & 5.92 & 20.00 & 9.44 & 5.33 & 20.00 \\
\hline & $L_{s}$ & - & $\mathbf{0}$ & $\mathbf{0}$ & 13.4819 & $\mathbf{0}$ & $\mathbf{0}$ & 12.8602 \\
\hline \multirow{6}{*}{ GRC-LRAV } & \multirow{5}{*}{$P_{g}$} & 1 & 229.57 & 228.78 & 206.11 & 230.00 & 223.11 & 207.19 \\
\hline & & 2 & 5.00 & 0.00 & 0.00 & 1.11 & 0.00 & 0.00 \\
\hline & & 3 & 17.33 & 20.33 & 23.33 & 24.00 & 27.00 & 30.00 \\
\hline & & 6 & 0.20 & 0.00 & 0.00 & 0.00 & 0.00 & 0.00 \\
\hline & & 8 & 6.89 & 4.89 & 6.89 & 4.89 & 2.89 & 4.89 \\
\hline & $L_{s}$ & - & $\mathbf{0}$ & $\mathbf{0}$ & 23.6677 & $\mathbf{0}$ & $\mathbf{0}$ & 16.9111 \\
\hline LRAV & $\Delta \boldsymbol{P}_{D}$ & $\begin{array}{c}2 \\
3 \\
4 \\
5 \\
6 \\
9 \\
10 \\
11 \\
12 \\
13 \\
14 \\
\end{array}$ & $\begin{array}{c}\text { Eleven } \\
\text { zeros }\end{array}$ & $\begin{array}{c}\text { Eleven } \\
\text { zeros }\end{array}$ & $\begin{array}{c}-10.8918 \\
38.553 \\
-23.9922 \\
-3.6688 \\
0 \\
0 \\
0 \\
0 \\
0 \\
0 \\
0 \\
\end{array}$ & $\begin{array}{c}\text { Eleven } \\
\text { zeros }\end{array}$ & $\begin{array}{l}\text { Eleven } \\
\text { zeros }\end{array}$ & $\begin{array}{c}-10.8500 \\
38.4047 \\
-23.9000 \\
-3.6547 \\
0 \\
0 \\
0 \\
0 \\
0 \\
0 \\
0 \\
\end{array}$ \\
\hline GRC-LRAV & $\Delta \boldsymbol{P}_{D}$ & $\begin{array}{l}2 \\
3 \\
4 \\
\end{array}$ & $\begin{array}{c}\text { Eleven } \\
\text { zeros }\end{array}$ & $\begin{array}{c}\text { Eleven } \\
\text { zeros }\end{array}$ & $\begin{array}{l}-10.8918 \\
38.553 \\
-23.9922 \\
\end{array}$ & $\begin{array}{c}\text { Eleven } \\
\text { zeros }\end{array}$ & $\begin{array}{l}\text { Eleven } \\
\text { zeros }\end{array}$ & $\begin{array}{c}-10.8500 \\
38.4047 \\
-23.9000 \\
\end{array}$ \\
\hline
\end{tabular}




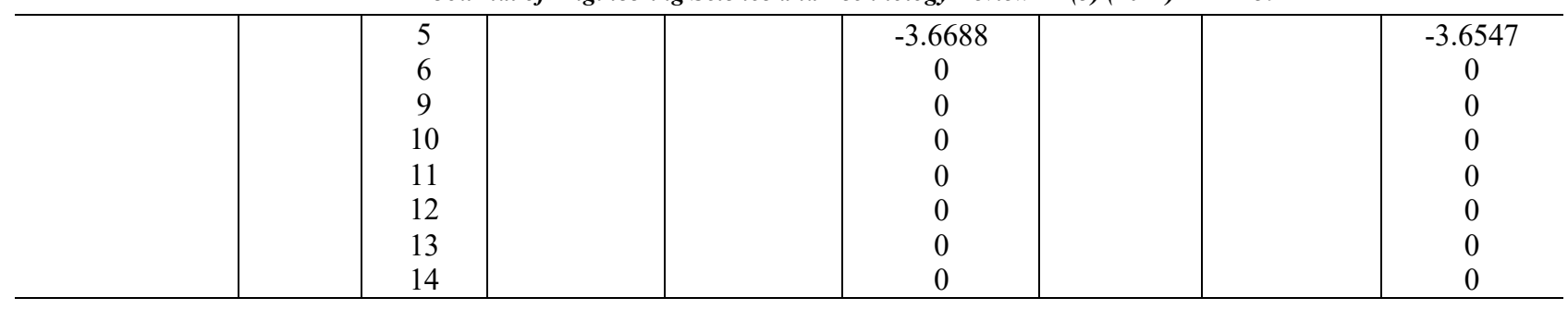

Table 4. Operational cost and load shedding due to LRA and GRC-LRA at fixed ramp rates in low load variation

\begin{tabular}{|c|c|c|c|c|c|c|c|c|}
\hline \multirow{3}{*}{$\begin{array}{c}\text { Attack } \\
\text { Resources, } R\end{array}$} & \multicolumn{4}{|c|}{ Operational Cost, \$/MWh } & \multicolumn{4}{|c|}{ Load Shedding, MW } \\
\hline & \multicolumn{2}{|c|}{ 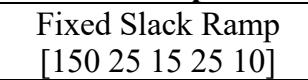 } & \multicolumn{2}{|c|}{$\begin{array}{l}\text { Fixed Tight Ramp [ }\left[\begin{array}{lll}30 & 5 & 3 \\
5 & 2\end{array}\right] \\
\end{array}$} & \multicolumn{2}{|c|}{$\begin{array}{l}\text { Fixed Slack Ramp } \\
{\left[\begin{array}{lllll}150 & 25 & 15 & 25 & 10\end{array}\right]}\end{array}$} & \multicolumn{2}{|c|}{$\begin{array}{l}\text { Fixed Tight Ramp [30 } 5 \\
35 \text { 2 }\end{array}$} \\
\hline & LRA & $\begin{array}{l}\text { GRC- } \\
\text { LRA }\end{array}$ & LRA & GRC-LRA & LRA & $\begin{array}{l}\text { GRC- } \\
\text { LRA }\end{array}$ & LRA & GRC-LRA \\
\hline$[0 ; 0 ; 0 ; 0 ; 0 ; 0]$ & 33638.52 & 33638.52 & 33638.52 & 33653.13 & 0 & $\begin{array}{l}0 \\
0\end{array}$ & 0 & $\begin{array}{c}0 \\
5780\end{array}$ \\
\hline$[0 ; 0 ; 20 ; 0 ; 0 ; 20]$ & 36567.46 & 36671.8 & 36567.46 & 37523.68 & 26.3420 & 29.2941 & 26.3420 & 40.5788 \\
\hline
\end{tabular}

Economic loss only due to attack=Operational cost with $R$ in LRA - Operational cost without $R$ in LRA $\Rightarrow$ $36567.46-33638.52=2928.94$

Economic loss only due to ramp=Operational cost without $R$ in GRC-LRA- Operational cost without $R$ in LRA $\Rightarrow 33653.13-33638.52=14.61$

Economic loss due to attack and ramp separately= Economic loss only due to attack + Economic loss only due to ramp $\Rightarrow 2928.94+14.61=2943.55$
Economic loss due to both attack and ramp in GRC-LRA= Operational cost with $R$ in GRC-LRA- Operational cost without $R$ in LRA $\Longrightarrow 37523.68-33638.52=3885.16$

From the above economic loss analysis, it is clear that the economic loss in GRC-LRAV regarding scenario-1 is $3885.16 \$$ MWh and sum of economic loss due to ramp and attack individually is $2543.55 \$ \mathrm{MWh}$ as shown in Tab.5 and Fig.3. So, economic loss in GRC-LRAV is $1341.223 \$ / \mathrm{MWh}$ greater than the summation of economic losses due to LRAV and ramp.

Table 5. Economic loss due to Ramp, LRA and GRC-LRA at fixed ramp rates in low load variation

\begin{tabular}{c|c|c|c|c}
\hline \multirow{2}{*}{ Ramp Limits } & \multicolumn{4}{|c}{ Economic Loss, \$/MWh } \\
\cline { 2 - 5 } & Ramp & LRA & Ramp and LRA & GRC-LRA \\
\hline Fixed Slack Ramp & 0 & 2928.938 & 2928.938 & 3033.286 \\
Fixed Tight Ramp & 14.612 & 2928.938 & 2543.542 & 3885.161 \\
\hline
\end{tabular}

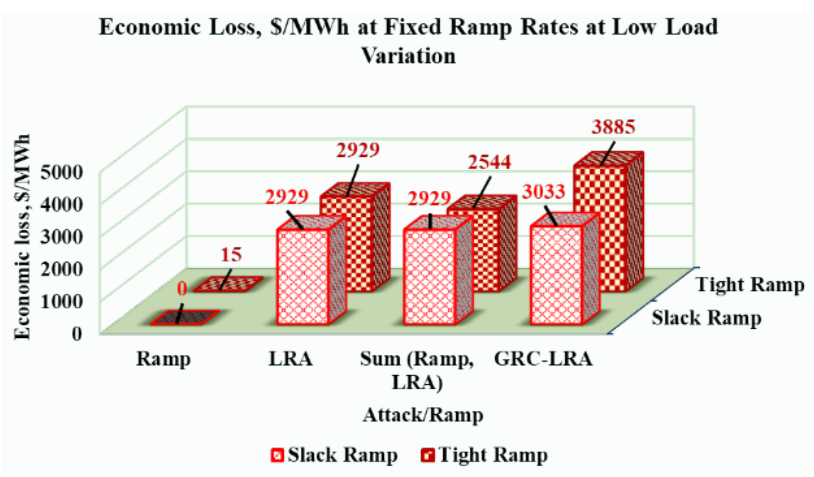

Fig. 3. Comparison of economic loss w.r.t. Ramp, LRA, $\sum$ Ramp, LRA and GRC-LRA at fixed ramp rates in low load variation

\subsubsection{Scenario-2: High Load Variation with $T=24$ at Slack and Tight Fixed Ramp Rates}

Let the load changes and attack triggering within 24 time steps is as given in Tab.6. Let the slack and tight ramp rates are $R_{-} u_{g}=R_{-} d_{g}=\left[\begin{array}{lllll}150 & 25 & 15 & 25 & 10\end{array}\right]$ and $R_{-} u_{g}=R_{-} d_{g}=\left[\begin{array}{lll}60 & 10\end{array}\right.$ $\left.\begin{array}{lll}6 & 10 & 4\end{array}\right]$ respectively. It is assumed that generators power

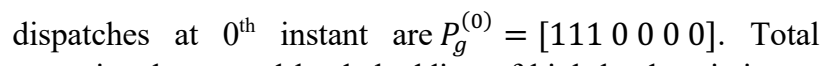
operational cost and load shedding of high load variation at fixed slack and tight ramp rates in 24 time steps of LRA and GRC-LRA are given in Tab.7.

From Tab.7 economic loss calculations are done which are given in Tab.8. The economic loss in GRC-LRAV at slack

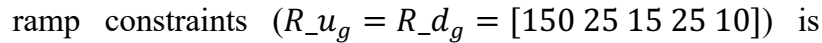
$5480.489 \$ / \mathrm{MWh}$ and economic loss due to ramp and attack separately is $4317.856 \$ / \mathrm{MWh}$ as shown in Tab.8. So, economic loss in GRC-LRA at fixed slack ramp rate in high load varying conditions is $4211.243 \$$ /MWh greater than the sum of economic losses of attack and ramp individually. From Tab.8 it is also clear that the economic loss in GRC-LRAV at

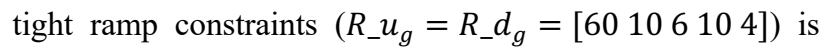
8755.397\$/MWh and economic loss due to ramp and attack separately is $4544.154 \$$ /MWh as shown in Tab.8 and Fig.4. So, economic loss in GRC-LRA is $4211.243 \$ / \mathrm{MWh}$ greater than the sum of economic losses due to attack and ramp individually.

Table 6. Load changes and attack triggering within 24 time steps

\begin{tabular}{c|c|c|c|c|c|c} 
Time Instances & 1 & 2 & 3 & 4 & 5 & 6 \\
Load & 148 & 173 & 220 & 244 & 259 & 248 \\
$\boldsymbol{R}$ & 0 & 0 & 0 & 0 & 20 & 20 \\
Time Instances & 7 & 8 & 9 & 10 & 11 & 12 \\
Load & 227 & 202 & 176 & 134 & 100 & 130 \\
\hline
\end{tabular}


Kommoju C Sravanthi and Mercy Rosalina Kotapuri/

Journal of Engineering Science and Technology Review 14 (3) (2021) 124 - 139

\begin{tabular}{|c|c|c|c|c|c|c|}
\hline $\boldsymbol{R}$ & 0 & 0 & 0 & 0 & 0 & 0 \\
\hline Time Instances & 13 & 14 & 15 & 16 & 17 & 18 \\
\hline Load & 157 & 168 & 195 & 225 & 244 & 241 \\
\hline $\boldsymbol{R}$ & 0 & 0 & 0 & 0 & 20 & 20 \\
\hline Time Instances & 19 & 20 & 21 & 22 & 23 & 24 \\
\hline Load & 230 & 210 & 176 & 157 & 138 & 103 \\
\hline $\boldsymbol{R}$ & 0 & 0 & 0 & 0 & 0 & 0 \\
\hline
\end{tabular}

Table 7. Operational cost and load shedding due to LRA and GRC-LRA at fixed ramp rates in High Load Variation

\begin{tabular}{|c|c|c|c|c|c|c|c|c|}
\hline \multirow{3}{*}{$\begin{array}{c}\text { Attack Resources, } \\
\mathbf{R}\end{array}$} & \multicolumn{4}{|c|}{ Operational Cost, $\$$ /MWh } & \multicolumn{4}{|c|}{ Load Shedding, MW } \\
\hline & \multicolumn{2}{|c|}{ 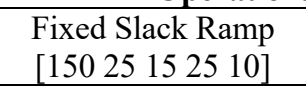 } & \multicolumn{2}{|c|}{$\begin{array}{l}\text { Fixed Tight Ramp [60 } \\
\left.\qquad \begin{array}{lllll}0 & 6 & 10 & 4\end{array}\right]\end{array}$} & \multicolumn{2}{|c|}{$\begin{array}{l}\text { Fixed Slack Ramp } \\
{\left[\begin{array}{lllll}150 & 25 & 15 & 25 & 10\end{array}\right]}\end{array}$} & \multicolumn{2}{|c|}{$\begin{array}{c}\text { Fixed Tight Ramp [60 } \\
106 \quad 6104]\end{array}$} \\
\hline & LRA & $\begin{array}{l}\text { GRC- } \\
\text { LRA }\end{array}$ & LRA & GRC-LRA & LRA & $\begin{array}{l}\text { GRC- } \\
\text { LRA }\end{array}$ & LRA & GRC-LRA \\
\hline $\begin{array}{l}{[0 ; 0 ; 0 ; 0 ; 0 ; 0 ; 0 ; 0 ; 0 ;} \\
0 ; 0 ; 0 ; 0 ; 0 ; 0 ; 0 ; 0 ; 0 ; 0 \\
\quad ; 0 ; 0 ; 0 ; 0 ; 0 ; 0]\end{array}$ & 91604.8 & 91604.8 & 91604.8 & 91831.09 & 0 & 0 & 0 & 0 \\
\hline $\begin{array}{c}{[0 ; 0 ; 0 ; 0 ; 20 ; 20 ; 0 ; 0} \\
0 ; 0 ; 0 ; 0 ; 0 ; 0 ; 0 ; 0 ; 20 \\
20 ; 0 ; 0 ; 0 ; 0 ; 0 ; 0]\end{array}$ & $\begin{array}{l}95922.6 \\
5\end{array}$ & 97085.28 & 95922.65 & 100360.2 & 24.089 & 34.816 & 24.089 & 65.252 \\
\hline
\end{tabular}

Table 8. Economic loss due to Ramp, LRA and GRC-LRA at Fixed Ramp Rates in High Load Variation

\begin{tabular}{c|c|c|c|c}
\hline \multirow{2}{*}{ Ramp Limits } & \multicolumn{4}{|c}{ Economic Loss, \$/MWh } \\
\cline { 2 - 5 } & Ramp & LRA & Ramp and LRA & GRC-LRA \\
\hline Fixed Slack Ramp & 0 & 4317.856 & 4317.856 & 5480.489 \\
Fixed Tight Ramp & 226.298 & 4317.856 & 4544.154 & 8755.397 \\
\hline
\end{tabular}

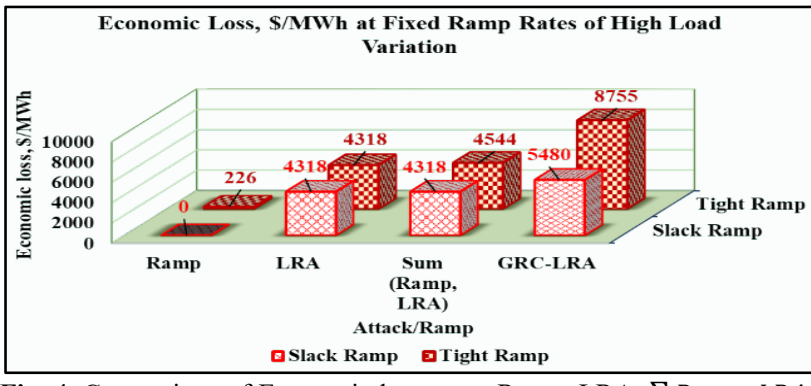

Fig. 4. Comparison of Economic loss w.r.t. Ramp, LRA, $\sum$ Ramp, LRA and GRC-LRA at fixed ramp rates of high load variation

\subsection{Economic Loss due to Most Damaging GRC-LRAV at Variable Ramp Rates}

\subsubsection{Scenario-1: Low Load Variation with $T=6$}

Similar to fixed ramp rates for the low load variation, the generation dispatches at $0^{\text {th }}$ instant are $P_{g}^{(0)}=\left[\begin{array}{lll}230 & 5.4114 .33\end{array}\right.$ $08.27]$.

\subsubsection{Slack Variable Ramp Rates}

In this article, most damaging GRC-LRAV is obtained at the variable ramp rates in all time instances. Variable ramp rates are simulated in random within the \pm 10 tolerance of fixed ramp rates i.e., slack fixed ramp rates $\left(R_{-} u_{g}=R_{-} d_{g}=[150\right.$ $25152510]$ ) are considered and the slack variable ramp rates are generated such that $\left[\begin{array}{lllll}135 & 22.5 & 13.5 & 2.5 & 9\end{array}\right] \leq$ $R_{u_{g}}$ (or) $R_{-} d_{g} \leq\left[\begin{array}{lllll}165 & 27.5 & 16.5 & 27.5 & 11\end{array}\right]$. The considered variable slack ramp rates at low load variation are presented in Tab.A1.

Total operational cost and load shedding of low load variation in 6 time steps at slack variable ramp rates in case of LRA and GRC-LRA are given in Tab.9.

From Tab.9 economic loss calculations are done which are given in Tab.10. It is clear that the economic loss in GRCLRAV at variable slack ramp rates given in Tab.A1 is 3067.124\$/MWh and economic loss due to ramp and attack separately is 2928.938\$/MWh as shown in Tab.10 and Fig.5. So, economic loss in GRC-LRA is $138.1861 \$$ /MWh greater than the summation individual loss by ramp and LRA.

\subsubsection{Tight Variable Ramp Rates}

Tight variable ramp rates are also simulated in random within the \pm 10 tolerance of fixed tight ramp rates $\left(R_{-} u_{g}=R_{-} d_{g}=\right.$

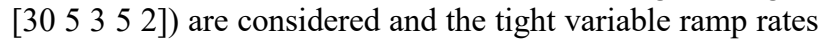
are generated such that $\left[\begin{array}{lllll}33 & 5.5 & 3.3 & 5.5 & 2.2\end{array}\right] \leq$

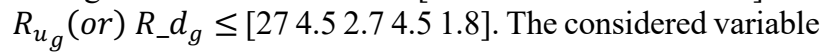
tight ramp rates at low load variation are presented in Tab.A2. Total operational cost and load shedding of low load variation in 6 time steps at tight variable ramp rates in case of LRA and GRC-LRA are given in Tab.9.

From Tab.9 economic loss calculations are done which are given in Tab.10. It is clear that the economic loss in GRCLRAV at variable tight ramp rates mentioned in Tab.A2 is 3882.804\$/MWh and economic loss due to ramp and attack separately is 2942.423\$/MWh as shown in Tab.10 and Fig.5. So, economic loss in GRC-LRA is $940.3808 \$ / \mathrm{MWh}$ greater than the summation individual loss by ramp and LRA. 
Kommoju C Sravanthi and Mercy Rosalina Kotapuri/

Journal of Engineering Science and Technology Review 14 (3) (2021) 124 - 139

Table 9. Operational cost and load shedding due to LRA and GRC-LRA at Variable ramp rates in Low Load Variation

\begin{tabular}{|c|c|c|c|c|c|c|c|c|}
\hline \multirow{3}{*}{$\begin{array}{c}\text { Attack } \\
\text { Resources, } R\end{array}$} & \multicolumn{4}{|c|}{ Operational Cost, \$/MWh } & \multicolumn{4}{|c|}{ Load Shedding, MW } \\
\hline & \multicolumn{2}{|c|}{ Variable Slack Ramp } & \multicolumn{2}{|c|}{ Variable Tight Ramp } & \multicolumn{2}{|c|}{$\begin{array}{c}\text { Variable Slack } \\
\text { Ramp }\end{array}$} & \multicolumn{2}{|c|}{ Variable Tight Ramp } \\
\hline & LRA & $\begin{array}{l}\text { GRC- } \\
\text { LRA }\end{array}$ & LRA & GRC-LRA & LRA & $\begin{array}{l}\text { GRC- } \\
\text { LRA }\end{array}$ & LRA & $\begin{array}{l}\text { GRC- } \\
\text { LRA }\end{array}$ \\
\hline$[0 ; 0 ; 0 ; 0 ; 0 ; 0]$ & 33638.52 & 33638.52 & 33658.52 & 33652.00 & 0 & 0 & 0 & 0 \\
\hline$[0 ; 0 ; 20 ; 0 ; 0 ; 20]$ & 36567.46 & 36705.64 & 36567.46 & 37521.32 & 26.3420 & 30.0018 & 26.3420 & 40.5374 \\
\hline
\end{tabular}

Table 10. Economic loss due to Ramp, LRA and GRC-LRA at Variable Ramp Rates in Low Load Variation

\begin{tabular}{c|c|c|c|c}
\hline Ramp Limits & \multicolumn{4}{|c}{ Economic Loss, \$/MWh } \\
\hline Name & Ramp & LRA & Ramp and LRA & GRC-LRA \\
\hline Variable Slack Ramp & 0 & 2928.938 & 2928.938 & 3067.124 \\
Variable Tight Ramp & 13.485 & 2928.938 & 2942.423 & 3882.804 \\
\hline
\end{tabular}

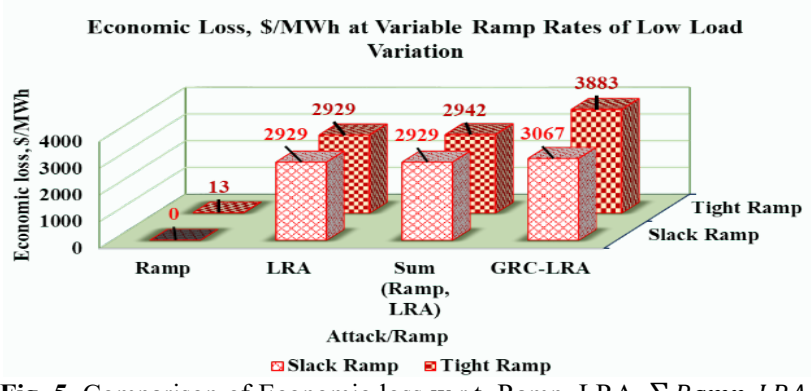

Fig. 5. Comparison of Economic loss w.r.t. Ramp, LRA, $\sum$ Ramp, LRA and GRC-LRA at variable ramp rates of low load variation

\subsubsection{Scenario-1: High Load Variation with $\boldsymbol{T}=\mathbf{2 4}$}

Similar to fixed ramp rates for the high load variation, the

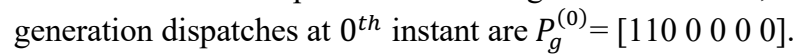

\subsubsection{Slack Variable Ramp Rates}

In this article, most damaging GRC-LRAV is obtained at the variable ramp rates in all time instances. Variable ramp rates are simulated in random within the \pm 10 tolerance of fixed ramp rates i.e., slack fixed ramp rates $\left(R_{-} u_{g}=R_{-} d_{g}=[150\right.$ $25152510])$ are considered and the slack variable ramp rates are generated such that $\left[\begin{array}{lllll}135 & 22.5 & 13.5 & 2.5 & 9\end{array}\right] \leq$
$R_{u_{g}}($ or $) R_{-} d_{g} \leq\left[\begin{array}{lllll}165 & 27.5 & 16.5 & 27.5 & 11\end{array}\right]$. The considered variable slack ramp rates at high load variation of 24 instances are presented in Tab.A3.

Total operational cost and load shedding of low load variation in 24 time steps at slack variable ramp rates in case of LRA and GRC-LRA are given in Tab.11.

From Tab.11 economic loss calculations are done which are given in Tab.12. It is clear that the economic loss in GRCLRAV at variable slack ramp rates represented in Tab.A3 is 5485.389\$/MWh and economic loss due to ramp and attack separately is $4317.856 \$$ MWh as shown in Tab.12 and Fig.5. So, economic loss in GRC-LRA is $1167.533 \$$ /MWh greater than the summation individual loss by ramp and LRA.

\subsubsection{Tight Variable Ramp Rates}

Tight variable ramp rates are also simulated in random within the \pm 10 tolerance of fixed tight ramp rates $\left(R_{-} u_{g}=R_{-} d_{g}=\right.$

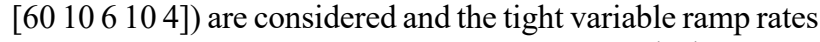

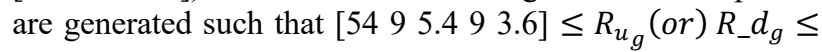
[66 1116.61114 .4 . The considered variable tight ramp rates at low load variation are presented in Tab.A4.

Total operational cost and load shedding of low load variation in 24 time steps at tight variable ramp rates in case of LRA and GRC-LRA are given in Tab.11.

Table 11. Operational cost and load shedding due to LRA and GRC-LRA at Variable ramp rates in High Load Variation

\begin{tabular}{c|c|c|c|c|c|c|c|c}
\hline \multirow{2}{*}{$\begin{array}{c}\text { Attack } \\
\text { Resources, }\end{array}$} & \multicolumn{4}{|c|}{ Operational Cost, \$/MWh } & \multicolumn{3}{c}{ Load Shedding, MW } \\
\cline { 2 - 9 } & Variable Slack Ramp & Variable Tight Ramp & \multicolumn{2}{c}{$\begin{array}{c}\text { Variable Slack } \\
\text { Ramp }\end{array}$} & \multicolumn{2}{c}{ Variable Tight Ramp } \\
\cline { 2 - 8 } & LRA & $\begin{array}{c}\text { GRC- } \\
\text { LRA }\end{array}$ & LRA & GRC-LRA & LRA & $\begin{array}{c}\text { GRC- } \\
\text { LRA }\end{array}$ & LRA & $\begin{array}{c}\text { GRC- } \\
\text { LRA }\end{array}$ \\
\hline $\begin{array}{c}{[0 ; 0 ; 0 ; 0 ; 0 ; 0 ; 0 ; 0 ;} \\
0 ; 0 ; 0 ; 0 ; 0 ; 0 ; 0 ; 0 ; 0 ; \\
0 ; 0 ; 0 ; 0 ; 0 ; 0 ; 0 ; 0] \\
0 ; 0 ; 0 ; 0 ; 20 ; 20 ; 0 ; \\
0 ; 0 ; 0 ; 0 ; 0 ; 0 ; 0 ; 0 ; 0 ; \\
\begin{array}{c}20 ; 20 ; 0 ; 0 ; 0 ; 0 ; 0 ; 0 \\
]\end{array}\end{array}$ & 91604.8 & 91604.8 & 91604.8 & 91832.43 & 0 & 0 & 0 & 0 \\
\hline
\end{tabular}

From Tab.11 economic loss calculations are done which are given in Tab.12. It is clear that the economic loss in GRCLRAV at variable tight ramp rates given in Table.A4 is 8749.746 $\$$ MWh and economic loss due to ramp and attack separately is $4545.491 \$ / \mathrm{MWh}$ as shown in Tab.12 and Fig.6. So, economic loss in GRC-LRA is $4204.255 \$ / \mathrm{MWh}$ greater than the summation individual loss by ramp and LRA as shown in Fig.6.

GRC-LRA's BPP is solved by using both KKT conditions based single level MILPP method and Benders decomposition method. Comparison of two solving methods is done in terms of operational cost, load shedding, economic 
loss and computational time at tight variable ramp rates as given in Tab.13. The difference between single level MILLP and Benders decomposition in terms of economic loss, load shedding is almost zero and the computational time for single level MILPP is more compared to Benders decomposition (less variable number in Benders decomposition) as shown in Tab.13.
Economic Loss, \$/MWh at Variable Ramp Rates of High Load Variation

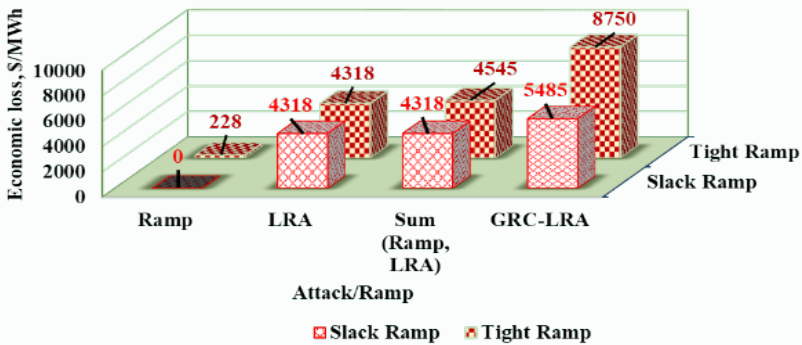

Fig. 6. Comparison of Economic loss w.r.t. Ramp, LRA, $\sum$ Ramp, LRA and GRC-LRA at variable ramp rates of high load variation

Table 12. Economic loss due to Ramp, LRA and GRC-LRA at Variable Ramp Rates in High Load Variation

\begin{tabular}{c|c|c|c|c}
\hline Ramp Limits & \multicolumn{4}{|c}{ Economic Loss, \$/MWh } \\
\hline Name & Ramp & LRA & Ramp and LRA & GRC-LRA \\
\hline $\begin{array}{c}\text { Variable Slack } \\
\text { Ramp } \\
\begin{array}{c}\text { Variable Tight } \\
\text { Ramp }\end{array}\end{array}$ & 0 & 4317.856 & 4317.856 & 5485.389 \\
\hline
\end{tabular}

Table 13. Comparison of Single-level MILPP and Benders decomposition methods in solving GRC-LRA at tight ramp rates of high load variation

\begin{tabular}{c|c|c|c}
\hline In GRC-LRA & Single level MILPP & Benders decomposition & Difference \\
\hline Operational cost, \$/MWh & 100354.542010487 & 100354.542010454 & 0 \\
Load Shedding, MW & 64.5967751310903 & 64.596775131090300 & 0 \\
Economic loss, \$/MWh & 8749.74640800931 & 8749.74640800925 & 0 \\
Computational Time, s & 2000.831727 & 333.507929 & - \\
\hline
\end{tabular}

4.3 Optimal Attack-Defense Strategy by Probabilistic Defense against GRC-LRA

An optimal attack-defense against GRC-LRAV is applied by finding optimal critical measuring units, then by framing possible attack strategies and further by playing static zerosum game. In this article, an optimal attack-defense strategy is represented for high load variation of the test system at tight variable ramp rates.

\subsubsection{Critical Measuring Units for Optimal Attack- Defense Strategy}

Let the attack resources of the test system operating at high load variation with $T=24$ and subjected to tight variable ramp rates as shown in Tab.A4. The economic loss due to GRC-LRAV in that case is $8749.746 \$ / \mathrm{MWh}$. Based on the algorithm given in Section-3.3, the critical measuring units are derived based on the rank of the measuring unit after defending only that specific unit, are shown in Tab.14.

Now select the units $P_{D_{6}}, P_{D_{5}}, P_{D_{9}}$ and $P_{D_{7}}$ (ranks of 1 to 4 ) and if defense is applied to all those four units at once, then the resultant economic loss $\cong 0$. Hence these units can be considered as critical measuring units.

\subsubsection{Probabilities of Optimal Attack-Defense Strategy of GRC-LRA}

As mentioned in section-3.3, among four critical units, let the attacker has access to three units and the remaining noncritical units (maximum attack resources of 20). Let the defender has access to one critical unit among four.

Then the maximum number of possible attack strategies be $4_{C_{3}}=4$ and maximum number of possible defense strategies is $4_{C_{3}}=4$ [26]. The possible attack and defense strategies with their corresponding economic loss is shown in Tab.15.

The probabilities of optimal attack-defense strategy against GRC-LRAV (at Nash equilibrium) are given in Tab.16.

Table 14. Rank of measuring unit after defending each unit

\begin{tabular}{c|c|c|c|c|c|c|c|c|c|c|c}
\hline $\begin{array}{c}\text { Measuri } \\
\text { ng Unit }\end{array}$ & $P_{D_{1}}$ & $P_{D_{2}}$ & $P_{D_{3}}$ & $P_{D_{4}}$ & $P_{D_{5}}$ & $P_{D_{6}}$ & $P_{D_{7}}$ & $P_{D_{8}}$ & $P_{D_{9}}$ & $P_{D_{10}}$ & $P_{D_{11}}$ \\
\hline $\begin{array}{c}\text { Econom } \\
\text { ic Loss } \\
\text { After }\end{array}$ & $\begin{array}{c}1195.1 \\
04\end{array}$ & $\begin{array}{c}1195.1 \\
04\end{array}$ & $\begin{array}{c}1195.1 \\
04\end{array}$ & $\begin{array}{c}1195.1 \\
04\end{array}$ & $\begin{array}{c}788.47 \\
27\end{array}$ & $\begin{array}{c}500.00 \\
59\end{array}$ & $\begin{array}{c}897.44 \\
89\end{array}$ & $\begin{array}{c}997.51 \\
69\end{array}$ & $\begin{array}{c}869.19 \\
98\end{array}$ & $\begin{array}{c}1088.7 \\
53\end{array}$ & $\begin{array}{c}1195.1 \\
04\end{array}$ \\
$\begin{array}{c}\text { Defense } \\
\text { Rank }\end{array}$ & 7 & 7 & 7 & 7 & 2 & 1 & 4 & 5 & 3 & 6 & 7 \\
\hline $\begin{array}{c}\text { Measuri } \\
\text { ng Unit }\end{array}$ & $P_{L_{1}}$ & $P_{L_{2}}$ & $P_{L_{3}}$ & $P_{L_{4}}$ & $P_{L_{5}}$ & $P_{L_{6}}$ & $P_{L_{7}}$ & $P_{L_{8}}$ & $P_{L_{9}}$ & $P_{L_{10}}$ & $P_{L_{11}}$ \\
\hline
\end{tabular}


Kommoju C Sravanthi and Mercy Rosalina Kotapuri/

Journal of Engineering Science and Technology Review 14 (3) (2021) 124 - 139

\begin{tabular}{|c|c|c|c|c|c|c|c|c|c|c|c|}
\hline $\begin{array}{c}\text { Econom } \\
\text { ic Loss } \\
\text { After } \\
\text { Defense } \\
\text { Rank }\end{array}$ & $\begin{array}{c}8749.7 \\
46 \\
20\end{array}$ & $\begin{array}{c}7353.7 \\
15 \\
15\end{array}$ & $\begin{array}{c}6757.7 \\
32 \\
11\end{array}$ & $\begin{array}{c}7454.8 \\
55 \\
\\
17\end{array}$ & $\begin{array}{c}8001.2 \\
95 \\
18\end{array}$ & $\begin{array}{c}7284.6 \\
31 \\
12\end{array}$ & $\begin{array}{c}6677.0 \\
91 \\
8\end{array}$ & $\begin{array}{c}6684.9 \\
05 \\
9\end{array}$ & $\begin{array}{c}7290.3 \\
13\end{array}$ & $\begin{array}{c}6699.1 \\
24 \\
10\end{array}$ & $\begin{array}{c}7427.4 \\
36 \\
16\end{array}$ \\
\hline $\begin{array}{l}\text { Measuri } \\
\text { ng Unit }\end{array}$ & $P_{L_{12}}$ & $P_{L_{13}}$ & $P_{L_{14}}$ & $P_{L_{15}}$ & $P_{L_{16}}$ & $P_{L_{17}}$ & $P_{L_{18}}$ & $P_{L_{19}}$ & $P_{L_{20}}$ & & \\
\hline $\begin{array}{c}\text { Econom } \\
\text { ic Loss } \\
\text { After } \\
\text { Defense } \\
\text { Rank }\end{array}$ & $\begin{array}{c}8749.7 \\
46 \\
20\end{array}$ & $\begin{array}{c}8749.7 \\
46 \\
20\end{array}$ & $\begin{array}{c}8749.7 \\
46 \\
20\end{array}$ & $\begin{array}{c}8749.7 \\
46 \\
20\end{array}$ & $\begin{array}{c}8749.7 \\
46 \\
20\end{array}$ & $\begin{array}{c}8044.6 \\
41 \\
19\end{array}$ & $\begin{array}{c}7348.2 \\
06 \\
14\end{array}$ & $\begin{array}{c}6757.7 \\
32 \\
11\end{array}$ & $\begin{array}{c}8749.7 \\
46 \\
20\end{array}$ & & \\
\hline
\end{tabular}

$\underline{\text { Table 15. Utility table, economic loss, } \$ / \mathrm{MWh} \text { of all possible attack and defense strategies }}$

\begin{tabular}{c|c|c|c|c}
\hline Strategies & $\boldsymbol{D}_{\mathbf{1}}=\boldsymbol{P}_{\boldsymbol{D}_{\mathbf{5}}}$ & $\boldsymbol{D}_{\mathbf{2}}=\boldsymbol{P}_{\boldsymbol{D}_{\mathbf{6}}}$ & $\boldsymbol{D}_{\mathbf{3}}=\boldsymbol{P}_{\boldsymbol{D}_{\mathbf{7}}}$ & $\boldsymbol{D}_{\mathbf{4}}=\boldsymbol{P}_{\boldsymbol{D}_{\mathbf{9}}}$ \\
\hline $\boldsymbol{A}_{\mathbf{1}}=\boldsymbol{P}_{\boldsymbol{D}_{\mathbf{5}}}, \boldsymbol{P}_{\boldsymbol{D}_{\mathbf{6}}}, \boldsymbol{P}_{\boldsymbol{D}_{\mathbf{9}}}$ & 435.1796 & 562.2581 & 227.6344 \\
$\boldsymbol{A}_{\mathbf{2}}=\boldsymbol{P}_{\boldsymbol{D}_{\mathbf{5}}}, \boldsymbol{P}_{\boldsymbol{D}_{\mathbf{7}}}, \boldsymbol{P}_{\boldsymbol{D}_{\mathbf{9}}}$ & 461.3374 & 500.0059 & 562.2581 \\
$\boldsymbol{A}_{\mathbf{3}}=\boldsymbol{P}_{\boldsymbol{D}_{\mathbf{5}}}, \boldsymbol{P}_{\boldsymbol{D}_{\mathbf{6}}}, \boldsymbol{P}_{\boldsymbol{D}_{\mathbf{7}}}$ & 227.6344 & 250.1581 & 227.6344 \\
$\boldsymbol{A}_{\mathbf{4}}=\boldsymbol{P}_{\boldsymbol{D}_{\mathbf{6}}}, \boldsymbol{P}_{\boldsymbol{D}_{\mathbf{7}}}, \boldsymbol{P}_{\boldsymbol{D}_{\mathbf{9}}}$ & 788.4727 & 461.3374 & 4351 \\
\hline
\end{tabular}

Table 16. Probabilities of optimal attack-defense strategy (Nash equilibrium) at variable tight ramp rates of high loading variation

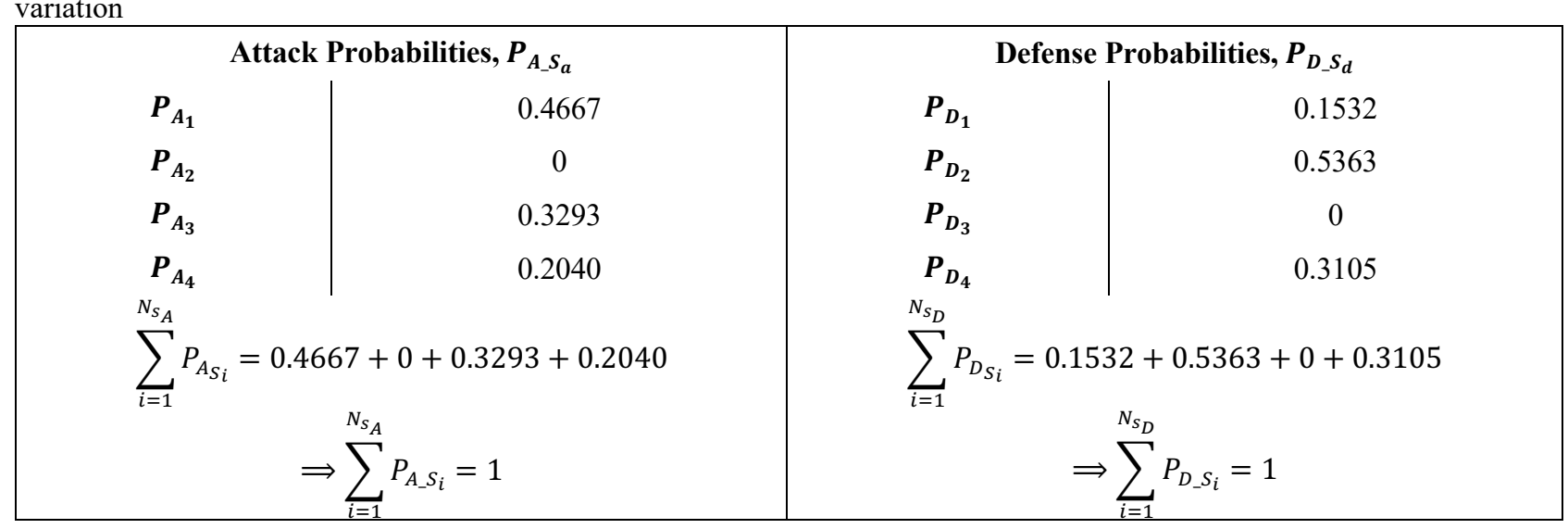

\section{Conclusions}

Cyber attacks in power systems are noteworthy as they can make system's economy, security and reliability vulnerable. Disruptive agents (attackers) can intrude into the system with complete or incomplete network information and inject undetectable false data into the system to mislead state estimation. Such kind of undetectable false data attacks are called FDIAs. LRAs are practical FDIAs that target bus power injections and line power flows to create unnecessary load shedding and line outages also that lead to considerable economic loss. To find the most damaging LRAV, a BPP is framed with attacker in upper level and basic SCOPF (operator) in the lower level. In this article generator ramp constraints are induced into basic SCOPF, to find the impact of ramp constraints on economic loss when an attack is triggered. The effect of ramp rates can directly effect a generator (when attacked) and create loss [23, 24]. The advantageous issue in this work is even if loads/lines are attacked in place of a generator, fixed/variable ramp rates can lead to more economic loss than basic LRA.

Mathematical model of GRC-LRA is framed on incorporating ramp constraints to basic LRA modelling. GRC-LRA's impact on operational cost/economic loss of the system is found by using two solvers. One is BPP is converted to KKT conditions based single-level MILPP method and the other is solving BPP directly by Benders decomposition method. GRC-LRA's effect is studied on the modified IEEE14 bus test system. To illustrate the impact of ramp constraints in a better way, load is considered in two modes in which one of them is low load variation with 6 time instances and the other is high load variation with 24 time instances. In each mode of operation, again fixed and variable ramp rates are considered, whereas in further in each fixed and variable ramp rates are considered as slack (not-tight) or tight. Economic loss in two loading modes at fixed slack, fixed tight, variable slack and variable tight ramp rates are elucidated in Tab.5 and Fig.3, Tab.8 and Fig.4, Tab.10 and Fig.5 and, Tab.12 and Fig. 6 respectively. From the aforementioned tables it is clear that the economic loss in GRC-LRA case is more than the sum of economic loss in ramp and LRA individually. From this study it can be concluded that generator ramp constraints must be considered while analyzing LRAs against power grid dynamically. For providing optimal defense, optimal critical measuring units are selected based on the ranking on measuring units shown in Tab.14. Probabilities of optimal attack-defense strategies are obtained by playing static zerosum game on the utility table given in Tab.15. The optimal probabilities are given in Tab.16. Hence, this research gives the in-sight of the impacts of optimal attacking and defending strategies at fixed and variable ramp rates induced into LRA on SCOPF in power systems. 


\section{Acknowledgement}

The authors like to show their sincere gratitude to the management, faculty and colleagues of Vignan's Foundation for Science, Technology and Research who have helped to carry out this research.
This is an Open Access article distributed under the terms of the Creative Commons Attribution License.

\section{References}

1. T. Zhang, Y. Wang, X. Liang, Z. Zhuang and W. Xu, Cyber attacks in cyber-physical power systems: A case study with GPRS-based SCADA systems, $29^{\text {th }}$ Chin. Control. and Decis. Conf. (CCDC), pp. 6847-6852 (2017) https://doi.org/10.1109/CCDC.2017.7978413

2. Y. Mo, T. H. J. Kim, K. Brancik, D. Dickinson, H. Lee, A. Perrig and B. Sinopoli, Cyber-physical security of a smart grid infrastructure, Proc. of the IEEE, 100(1), 195 (2011). https://doi.org/10.1109/CISS.2010

3. G. Liang, S. R. Weller, J. Zhao, F. Lu and Z. Y. Dong, The 2015 ukraine blackout: Implications for false data injection attacks, IEEE Transactions on Power Systems, 32(4), 3317 (2016). https://doi.org/10.1109/TPWRS.2016.2631891

4. K. Chatterjee, V. Padmini and S. A. Khaparde, Rev. of cyber-attacks on Power Syst. Oper., in 2017 IEEE Region 10 Symposium (TENSYMP), Cochin, India, pp. 1-6 (2017). https://doi.org/10.1109/TENCONSpring.2017.8070085

5. Y. Yang, K. McLaughlin, T. Littler, S. Sezer, Eul Gyu Im, Z. Q. Yao, B. Pranggono and H. F. Wang, Man-in-the-middle attack test-bed investigating cyber-security vulnerabilities in Smart Grid SCADA systems, in 2012 Int. Conf. on Sustain. Power Gener. and Supply (SUPERGEN-2012), Hangzhou, China, pp. 1-8 (2012). https://doi.org/10.1049/cp.2012.1831

6. Y. Shen, M. Fei and D. Du, Cyber security study for power systems under denial of service attacks, Transactions of the Institute of Measurement and Control 41(6), 1, (2017). https://doi.org/10.1177/0142331217709528

7. Y. Liu, P. Ning and M. K. Reiter, False Data Injection Attacks against State Estimation in Electric Power Grids, ACM Transactions on Information and System Security 14(1), 1, (2011). https://doi.org/10.1145/1653662.1653666

8. Y. Yuan, Z. Li and K. Ren, Modeling Load Redistribution Attacks in Power Systems, IEEE Transactions on Smart Grid, 2(2), 382, (2011). https://doi.org/10.1109/TSG.2011.2123925

9. Y. Yuan, Z. Li and K. Ren, Quantitative Analysis of Load Redistribution Attacks in Power Systems, IEEE Transactions on Parallel and Distribution Systems, 23(9), 1731, (2012). https://doi.org/10.1109/TPDS.2012.58

10. J. M. Arroyo and F. D. Galiana, On the Solution of the Bilevel Programming Formulation of the Terrorist Threat Problem, IEEE Transactions on Power Systems, 20(2), 789, (2005). https://doi.org/10.1109/TPWRS.2005.846198

11. X. Liu, Z. Li, Z. Shuai and Y. Wen, Cyber Attacks Against the Economic Operation of Power Systems: A Fast Solution, IEEE Transactions on Smart Grid 8(2), 1023, (2017). https://doi.org/10.1109/TSG.2016.2623983

12. D. Choeum and D. H. Choi, Vulnerability Assessment of Conservation Voltage Reduction to Load Redistribution Attack in Unbalanced Active Distribution Networks, IEEE Transactions on Industrial Informatics, (2020).

https://doi.org/10.1109/TII.2020.2980590

13. R. Kaviani and K. W. Hedman (2019) Identifying an Exploitable Structure for the Core Problem of Load-Redistribution Attack Problems, in 2019 North American Power Symposium (NAPS), Wichita, KS, USA, pp. 1-6 (2019). https://doi.org/10.1109/NAPS46351.2019.9000221

14. X. Liu and Z. Li, Local Load Redistribution Attacks in Power Systems with Incomplete Network Information, IEEE Transactions on Smart Grid 5(4), 1665, (2014). https://doi.org/10.1109/TSG.2013.2291661
15. X. Liu and Z. Li, Local Topology Attacks in Smart Grids. IEEE Transactions on Smart Grid, 8(6), 2617, (2017). https://doi.org/10.1109/TSG.2016.2532347

16. Y. Xiang, L. Wang, D. Yu and N. Liu, Coordinated attacks against power grids: Load redistribution attack coordinating with generator and line attacks, in 2015 IEEE Power \& Energy Society General Meeting, Denver, CO, USA, pp. 1-5 (2015). https://doi.org/10.1109/PESGM.2015.7286402

17. Y. Xiang, L. Wang and N. Liu, A framework for modeling load redistribution attacks coordinating with switching attacks, in 2017 IEEE Power \& Energy Soc. Gen. Meet., pp 1-5 (2018). https://doi.org/10.1109/PESGM.2017.8274621

18. Z. Li, M. Shahidehpour, Z. Alabdulwahab and A. Abusorrah, Bilevel model for analyzing coordinated cyber-physical attacks on power systems, IEEE Transactions on Smart Grid, 7(5), 2260, (2015). https://doi.org/10.1109/TSG.2015.2456107

19. Fu J, Wang L, Hu B, Xie K, Chao H, Zhou P, A Sequential Coordinated Attack Model for Cyber-Physical System Considering Cascading Failure and Load Redistribution in 2018 2nd IEEE Conference on Energy Internet and Energy System Integration (EI2), Beijing, China, pp. 1-6, (2018). https://doi.org/10.1109/EI2.2018.8582135

20. Y. Xiang, Z. Ding, Y. Zhang and L. Wang, Power system reliability evaluation considering load redistribution attacks, IEEE Trans on $\begin{array}{llll}\text { Smart } & \text { G(2), } & \text { 889, }\end{array}$ https://doi.org/10.1109/TSG.2016.2569589

21. Z. Ding, Y. Xiang and L. Wang (2016) Quantifying the influence of local load redistribution attack on power supply adequacy. In: 2016 IEEE Power and Energy Society General Meeting (PESGM), pp 15. Boston, MA, USA. https://doi.org/10.1109/PESGM.2016.7741526

22. H. Shayan and T. Amraee, Network Constrained Unit Commitment Under Cyber Attacks Driven Overloads, IEEE Transactions on Smart Grid, 10(6), 6449, (2019). https://doi.org/10.1109/TSG.2019.2904873

23. D. H. Choi and L. Xie, Ramp-induced data attacks on look-ahead dispatch in real-time power markets, IEEE Trans on Smart Grid, 4(3), 1235, (2013). https://doi.org/10.1109/TSG.2012.2228508

24. L. Che, X. Liu, Z. Shuai and J. Zhao, The Impact of Ramp-Induced Data Attacks on Power System Operational Security, IEEE Transactions on Industrial Informatics, 15(9), 5064, (2019).

25. K. B. Krishna, K. M. Rosalina, N. Ramaraj, Complete and Incomplete Observability Analysis by Optimal PMU Placement Techniques of a Network, Journal of Electrical Engineering \& Technology, 13(5), 1814, (2018). https://doi.org/10.5370/JEET.2018.13.5.1814

26. Y. Xiang and L. Wang, A game-theoretic approach to optimal defense strategy against load redistribution attack, in 2015 IEEE Power \& Energy Society General Meeting, pp. 1-5 (2015). https://doi.org/10.1109/PESGM.2015.7286529

27. Y. Xiang and L. Wang, A game-theoretic study of load redistribution attack and defense in power systems, Electrical Power Systems Research 151, 12, (2017) https://doi.org/10.1016/j.epsr.2017.05.020

28. R. D. Zimmerman, C. E. Murillo-Sánchez and R. J. Thomas, MATPOWER: Steady-state operations, planning, and analysis tools for power systems research and education, IEEE Transactions on Power Systems, 26(1), 12, (2011). https://doi.org/10.1109/TPWRS.2010.2051168

29. IBM ILOG CPLEX Optimization Studio. Version 12.9.0. Available: https://www.ibm.com/inen/products/ilogeplexoptimizationstudio

\section{Appendix}


Kommoju C Sravanthi and Mercy Rosalina Kotapuri/

Journal of Engineering Science and Technology Review 14 (3) (2021) 124 - 139

Tab.A1 and Tab.A2 represent the slack and tight variable ramp rates at low load variation $\forall t=\{1,2, \ldots, 6\}$ respectively. However, Tab.A3 and Tab.A4 represent the slack and tight variable ramp rates at high load variations $\forall t=\{1,2, \ldots, 24\}$ respectively.

Table A1. Slack Variable Ramp Rates at Low Load Variation of $T=6$

\begin{tabular}{l|c|c|c|c|c}
\hline & $\boldsymbol{P}_{\boldsymbol{g}_{\mathbf{1}}}$ & $\boldsymbol{P}_{\boldsymbol{g}_{\mathbf{2}}}$ & $\boldsymbol{P}_{\boldsymbol{g}_{\mathbf{3}}}$ & $\boldsymbol{P}_{\boldsymbol{g}_{\mathbf{6}}}$ & $\boldsymbol{P}_{\boldsymbol{g}_{\mathbf{8}}}$ \\
\hline $\boldsymbol{t}$ & 159 & 26.5 & 15.9 & 26.5 & 10.6 \\
$\boldsymbol{t}=\mathbf{2}$ & 162 & 27 & 16.2 & 27 & 10.8 \\
$\boldsymbol{t}=\mathbf{3}$ & 139.5 & 23.25 & 13.95 & 23.25 & 9.3 \\
$\boldsymbol{t}=\mathbf{4}$ & 162 & 27 & 16.2 & 27 & 10.8 \\
$\boldsymbol{t}=\mathbf{5}$ & 154.5 & 25.75 & 15.45 & 25.75 & 10.3 \\
$\boldsymbol{t}=\mathbf{6}$ & 138 & 23 & 13.8 & 23 & 9.2 \\
\hline
\end{tabular}

Table A2. Tight Variable Ramp Rates at Low Load Variation of $T=6$

\begin{tabular}{l|l|l|l|l|l}
\hline & \multicolumn{1}{|c|}{$\boldsymbol{P}_{\boldsymbol{g}_{\mathbf{1}}}$} & $\boldsymbol{P}_{\boldsymbol{g}_{\mathbf{2}}}$ & \multicolumn{1}{c|}{$\boldsymbol{P}_{\boldsymbol{g}_{\mathbf{3}}}$} & \multicolumn{1}{c}{$\boldsymbol{P}_{\boldsymbol{g}_{\mathbf{6}}}$} & $\boldsymbol{P}_{\boldsymbol{g}_{\mathbf{8}}}$ \\
\hline $\boldsymbol{t}$ & $30 \mathbf{1}$ & 5 & 3 & 5 & 2 \\
$\boldsymbol{t}$ & 29.7 & 4.95 & 2.97 & 4.95 & 1.98 \\
$\boldsymbol{t}=\mathbf{3}$ & 30.9 & 5.15 & 3.09 & 5.15 & 2.06 \\
$\boldsymbol{t}=\mathbf{4}$ & 31.2 & 5.2 & 3.12 & 5.2 & 2.08 \\
$\boldsymbol{t}=\mathbf{5}$ & 31.5 & 5.25 & 3.15 & 5.25 & 2.1 \\
$\boldsymbol{t}$ & 28.8 & 4.8 & 2.88 & 4.8 & 1.92 \\
\hline
\end{tabular}

Table A3. Slack Variable Ramp Rates at High Load Variation of $T=24$

\begin{tabular}{|c|c|c|c|c|c|}
\hline & $P_{g_{1}}$ & $\boldsymbol{P}_{g_{2}}$ & $P_{g_{3}}$ & $P_{g_{6}}$ & $P_{g_{8}}$ \\
\hline$t=1$ & 156 & 26 & 15.6 & 26 & 10.4 \\
\hline$t=2$ & 157.5 & 26.25 & 15.75 & 26.25 & 10.5 \\
\hline$t=3$ & 157.5 & 26.25 & 15.75 & 26.25 & 10.5 \\
\hline$t=4$ & 147 & 24.5 & 14.7 & 24.5 & 9.8 \\
\hline$t=5$ & 154.5 & 25.75 & 15.45 & 25.75 & 10.3 \\
\hline$t=6$ & 139.5 & 23.25 & 13.95 & 23.25 & 9.3 \\
\hline$t=7$ & 156 & 26 & 15.6 & 26 & 10.4 \\
\hline$t=\mathbf{8}$ & 136.5 & 22.75 & 13.65 & 22.75 & 9.1 \\
\hline$t=9$ & 144 & 24 & 14.4 & 24 & 9.6 \\
\hline$t=10$ & 136.5 & 22.75 & 13.65 & 22.75 & 9.1 \\
\hline$t=11$ & 138 & 23 & 13.8 & 23 & 9.2 \\
\hline$t=12$ & 159 & 26.5 & 15.9 & 26.5 & 10.6 \\
\hline$t=13$ & 156 & 26 & 15.6 & 26 & 10.4 \\
\hline$t=14$ & 144 & 24 & 14.4 & 24 & 9.6 \\
\hline$t=15$ & 163.5 & 27.25 & 16.35 & 27.25 & 10.9 \\
\hline$t=16$ & 136.5 & 22.75 & 13.65 & 22.75 & 9.1 \\
\hline$t=17$ & 148.5 & 24.75 & 14.85 & 24.75 & 9.9 \\
\hline$t=18$ & 147 & 24.5 & 14.7 & 24.5 & 9.8 \\
\hline$t=19$ & 157.5 & 26.25 & 15.75 & 26.25 & 10.5 \\
\hline$t=20$ & 159 & 26.5 & 15.9 & 26.5 & 10.6 \\
\hline$t=21$ & 141 & 23.5 & 14.1 & 23.5 & 9.4 \\
\hline$t=22$ & 150 & 25 & 15 & 25 & 10 \\
\hline$t=23$ & 148.5 & 24.75 & 14.85 & 24.75 & 9.9 \\
\hline$t=24$ & 154.5 & 25.75 & 15.45 & 25.75 & 10.3 \\
\hline
\end{tabular}

Table A4. Tight Variable Ramp Rates at High Load Variation of $T=24$

\begin{tabular}{|c|c|c|c|c|c|}
\hline & $P_{g_{1}}$ & $P_{g_{2}}$ & $P_{g_{3}}$ & $P_{g_{6}}$ & $P_{g_{8}}$ \\
\hline$t=1$ & 56.4 & 9.4 & 5.64 & 9.4 & 3.76 \\
\hline$t=2$ & 58.2 & 9.7 & 5.82 & 9.7 & 3.88 \\
\hline$t=3$ & 64.8 & 10.8 & 6.48 & 10.8 & 4.32 \\
\hline$t=4$ & 58.8 & 9.8 & 5.88 & 9.8 & 3.92 \\
\hline$t=5$ & 60.6 & 10.1 & 6.06 & 10.1 & 4.04 \\
\hline$t=6$ & 65.4 & 10.9 & 6.54 & 10.9 & 4.36 \\
\hline$t=7$ & 63.6 & 10.6 & 6.36 & 10.6 & 4.24 \\
\hline$t=8$ & 61.2 & 10.2 & 6.12 & 10.2 & 4.08 \\
\hline$t=9$ & 61.8 & 10.3 & 6.18 & 10.3 & 4.12 \\
\hline$t=10$ & 59.4 & 9.9 & 5.94 & 9.9 & 3.96 \\
\hline
\end{tabular}


Kommoju C Sravanthi and Mercy Rosalina Kotapuri/

Journal of Engineering Science and Technology Review 14 (3) (2021) 124 - 139

\begin{tabular}{|c|c|c|c|c|c|}
\hline$t=11$ & 57 & 9.5 & 5.7 & 9.5 & 3.8 \\
\hline$t=12$ & 55.8 & 9.3 & 5.58 & 9.3 & 3.72 \\
\hline$t=13$ & 60.6 & 10.1 & 6.06 & 10.1 & 4.04 \\
\hline$t=14$ & 59.4 & 9.9 & 5.94 & 9.9 & 3.96 \\
\hline$t=15$ & 65.4 & 10.9 & 6.54 & 10.9 & 4.36 \\
\hline$t=16$ & 63.6 & 10.6 & 6.36 & 10.6 & 4.24 \\
\hline$t=17$ & 57 & 9.5 & 5.7 & 9.5 & 3.8 \\
\hline$t=18$ & 64.8 & 10.8 & 6.48 & 10.8 & 4.32 \\
\hline$t=19$ & 55.2 & 9.2 & 5.52 & 9.2 & 3.68 \\
\hline$t=20$ & 55.8 & 9.3 & 5.58 & 9.3 & 3.72 \\
\hline$t=21$ & 63.6 & 10.6 & 6.36 & 10.6 & 4.24 \\
\hline$t=22$ & 58.8 & 9.8 & 5.88 & 9.8 & 3.92 \\
\hline$t=23$ & 63.6 & 10.6 & 6.36 & 10.6 & 4.24 \\
\hline$t=24$ & 61.8 & 10.3 & 6.18 & 10.3 & 4.12 \\
\hline
\end{tabular}

\section{Nomenclature}

Abbreviations

PMU

RTU

DoS attacks

SCADA

FDIA

FDIAV

SCED

SCOPF

LRA

LRAV

GRC-LRA

GRC-LRAV

BPP

MILPP

KKT conditions

\section{Symbols}

$P_{g_{i}}$

$L_{s_{k}}$

$\Delta P_{D_{k}}$

$\Delta P_{L_{l}}$

$\boldsymbol{R}$

$\theta_{D_{k}}$

$\theta_{D+k}$

$\theta_{D-k}$

$\theta_{L_{l}}$

$\theta_{L+l}$

$\theta_{L-l}$

$P_{D_{k}}$

$P_{L_{l}}$

$R \_u_{g_{i}}, R \_d_{g_{i}}$

$\boldsymbol{S F}, \boldsymbol{K D}$

$M, \varepsilon$

$\tau$

$\underline{A}_{l}, \bar{A}_{l}$

$\underline{B}_{i}, \bar{B}_{i}$

$\underline{\Gamma}_{k}, \bar{\Gamma}_{k}$
Phasor Measurement Unit

Remote Terminal Unit

Denial of Service attacks

Supervisory Control and Data Acquisition

False Data Injection Attack

False Data Injection Attack Vector

Security Constrained Economic Dispatch

Security Constrained Optimal Power Flow

Load Redistribution Attack

Load Redistribution Attack Vector

Generator Ramp Constraints induced Load Redistribution Attack

Generator Ramp Constraints induced Load Redistribution Attack Vector

Bi-level Programming Problem

Mixed Integer Linear Programming Problem

Karush-Kuhn-Tucker conditions

Power Dispatch of $i^{\text {th }}$ generator

Load Shedding/Curtailment of $k^{\text {th }}$ load

Load attack on $k^{\text {th }}$ load

Line attack on $l^{\text {th }}$ generator

Vector of attack resources

$\left\{1\right.$ if $\Delta P_{D_{k}} \neq 0$

\{ else 0

$\left\{1\right.$ if $\Delta P_{D_{k}}>0$

\{ else 0

$\left\{1\right.$ if $\Delta P_{D_{k}}<0$

\{ else 0

$\left\{1\right.$ if $\Delta P_{L_{l}} \neq 0$

ise 0

$\left\{1\right.$ if $\Delta P_{L_{l}}>0$

ifse 0

$\left\{1\right.$ if $\Delta P_{L_{l}}<0$

\{ else 0

Load demand of $k^{\text {th }}$ load

Power flow on $l^{\text {th }}$ line

Up and down ramp rates of $i^{\text {th }}$ generator

Shift Factor and Bus-generator incidence matrices

Sufficiently large and sufficiently small positive numbers

Attack deviation bound on load bus

Lagrange multipliers for upper and lower bounds of $l^{\text {th }}$ transmission line

Lagrange multipliers for upper and lower bounds of $i^{\text {th }}$ generator

Lagrange multipliers for upper and lower bounds of $k^{\text {th }}$ load 
Kommoju C Sravanthi and Mercy Rosalina Kotapuri/

Journal of Engineering Science and Technology Review 14 (3) (2021) 124 - 139

$r u_{i}^{t}, r d_{i}^{t}$

$\omega_{\underline{A}, l}, \omega_{\bar{A}, l}$,

$\omega_{\underline{B}, i}, \omega_{\bar{B}, i}$,

$\omega_{\Gamma, k}, \omega_{\overline{\Gamma, k}}$,

$\omega_{r u_{i}^{t}}, \omega_{r d_{i}^{t}}$

$A_{m}, D_{n}$

$P_{A_{m}}, P_{D_{n}}$

Lagrange multipliers for ramp up and ramp down of $i^{\text {th }}$ generator

Binary variables that represent complementary slackness conditions of $l^{\text {th }}$ transmission line, $i^{\text {th }}$ generator, $k^{\text {th }}$ load and ramping of $i^{\text {th }}$ generator

$m$ attack and $n$ defense

strategies

Probabilities of $m^{\text {th }}$ attack and $n^{\text {th }}$ defense strategies 